\title{
Investigation on the Flow Behavior of Side Channel Pumps Based on Vortex Identification
}

\author{
Fan Zhang ${ }^{1 *} \mathbb{D}$, Desmond Appiah ${ }^{1,2^{*}}$, Ke Chen $^{1}$, Shouqi Yuan ${ }^{1}$, Kofi Asamoah Adu-Poku ${ }^{1}$ and Lufeng Zhu ${ }^{1}$
}

\begin{abstract}
The momentum flow exchange between the impeller and side channel produces highly turbulent flows in side channel pumps. The turbulent flows feature complex patterns of vortex structures that are partly responsible for the dissipation of energy losses and unsteady pressure pulsations. The concept of turbulent flows in side channel pumps requires a reliable vortex identification criterion to capture and predict the effects of the vortex structures on the performance. For this reason, the current study presents the application of the new $\Omega$-criterion to a side channel pump model in comparison with other traditional methods such as $Q$ and $\lambda_{2}$ criteria. The 3D flow fields of the pump were obtained through unsteady Reynolds-averaged Navier-Stokes (RANS) simulations. Comparative studies showed that the $\Omega$-criterion identifies the vortex of different intensities with a standard threshold, $\Omega=0.52$. The $Q$ and $\lambda_{2}$ criteria required different thresholds to capture vortex of different intensities thus leads to subjective errors. Comparing the $\Omega$-criterion intensity on different planes with the entropy losses and pressure pulsation, the longitudinal vortex plays an important role in the momentum exchange development which increases the head performance of the pump. However, the rate of exchange is impeded by the axial and radial vortices restricted in the impeller. Therefore, the impeller generates the highest entropy loss and pressure pulsation intensities which lower the output efficiency. Finally, the findings provide a fundamental background to the morphology of the vortex structures in the turbulent flows which can be dependent upon for efficiency improvement of side channel pumps.
\end{abstract}

Keywords: Side channel pumps, $\Omega$-criterion, Vortex, Entropy loss

\section{Introduction}

Description of the evolution of vortex structures explains the flow theory behind turbulent flows. Thus, vortex structure identification has been extensively identified and studied not only to model the turbulence but reveal turbulence production and dissipation in turbomachines. To date, there exist no general identification method of vortex structures and the available methods are case dependent [1]. Most of the commonly used identification methods in turbomachinery depend on a key parameter called the velocity gradient tensor. The velocity gradient tensor $(\nabla \vec{V})$ can be defined as:

\footnotetext{
*Correspondence: fzhang@ujs.edu.cn; bembo88donk@yahoo.com

${ }^{1}$ National Research Center of Pumps, Jiangsu University,

Zhenjiang 212013, China

Full list of author information is available at the end of the article
}

$$
\lambda^{3}+P \lambda^{2}+Q \lambda+R=0,
$$

where $P, Q$ and $R$ are the invariants of the velocity gradient tensor. The eigenvalues, $\lambda$ of $(\nabla \vec{V})$ is determined by calculating the cubic characteristic polynomial, $\operatorname{det}(\nabla \vec{V}-\lambda I)=0$, here $I$ is the identity matrix.

Additionally, $(\nabla \vec{V})$ can be decomposed into a symmetric part, $A$ and antisymmetric part, $B$.

$$
\nabla \vec{V}=1 / 2\left(\nabla \vec{V}+\nabla \vec{V}^{\mathrm{T}}\right)+1 / 2\left(\nabla \vec{V}-\nabla \vec{V}^{\mathrm{T}}\right)=A+B .
$$

The popular Q-criterion proposed by Hunt et al. [2] identifies vortex as a fluid region with a positive second invariant $Q$ of the velocity gradient tensor $(\nabla \vec{V})$ for incompressible flows. $Q$-criterion can be expressed as the difference between the magnitude of the rate of shear 
strain and vorticity magnitude. Vortex structures evolve around region of $Q>0$.

$$
Q=1 / 2\left(\|B\|^{2}-\|A\|^{2}\right),
$$

where, $\|\bullet\|$ denote the Frobenius norm and the $\nabla \vec{V}$ is given as $\nabla \vec{V}=\left(\begin{array}{lll}\frac{\partial u}{\partial x} & \frac{\partial u}{\partial y} & \frac{\partial u}{\partial z} \\ \frac{\partial v}{\partial x} & \frac{\partial v}{\partial y} & \frac{\partial v}{\partial z} \\ \frac{\partial w}{\partial x} & \frac{\partial w}{\partial y} & \frac{\partial w}{\partial z}\end{array}\right)$.

Furthermore, Jeong and Hussain [3] postulated the $\lambda_{2}$-criterion which also depends on $(\nabla \vec{V})$. This method seems to reduce the pressure but removes the effects of unsteady strain and viscosity by taking the gradient of the incompressible Navier-Stokes equation. The equation decomposes into two parts $A$ and $B$ representing symmetric part and antisymmetric part respectively.

$$
A^{2}+B^{2}=-\frac{1}{\rho} \nabla^{2} p
$$

Concerning the $\lambda_{2}$-criterion, vortex exists in the domain where there are two negative eigenvalues of $A^{2}+B^{2}$ i.e. $\lambda_{1} \geq \lambda_{2} \geq \lambda_{3}, \lambda_{2}<0$. The relation between negative eigenvalues of $A^{2}+B^{2}$ and $Q$ can be expressed as:

$$
Q=-1 / 2 \operatorname{tr}\left(A^{2}+B^{2}\right)=-1 / 2\left(\lambda_{1}+\lambda_{2}+\lambda_{3}\right) .
$$

This method depends on the minimum value of $\lambda_{2}$ extracted to represent the vortex core position. The vortex core lines revealed that using this method usually are not continuous because they are composed of several vortex structure segments.

To capture the vortex structures in turbulent flows, the $Q$ and $\lambda_{2}$ criteria have been commonly used. Wang et al. [4] analyzed the instantaneous vortical structures depicted by the vortex core region using the $\lambda_{2}$-criterion for different turbulence models in side channel pump. They concluded that the evolution of most vortical structures in the circumferential direction are responsible for the process of fluid exchange. Minakov et al. [5] showed the typical vortex structures in the operation of Francis turbines behind the runner using the $Q$-criterion. It is revealed that large vortices are found behind the runner and are the major source of the pressure pulsations. Asim et al. [6] depicted the variations in the downstream of the tongue region of a centrifugal pump. They indicated that the positive $Q$ - criterion values correspond to higher vorticity magnitude than the shear strain-rate. Furthermore, it was pointed out that the vortical structures formed downstream of the tongue region, and their strength decreases as the distance from the tongue increases.
Other notable literature on the application of these methods in different turbomachines can be found in Refs. [7-12]. However, these methods can be challenging in the identification of the actual vortical rotational motion due to their dependence on the threshold value selected. In other words, inappropriate thresholds misidentify vortex structures which do not produce accurate information on the inner flow dynamics. To solve this problem, Liu et al. [13] recently put forward a method of vortex identification based on the decomposing of $(\nabla \vec{V})$ into a vortical part and non-vortical part and introduced a parameter called $\Omega$. As stated in Eq. (2), $A=1 / 2\left(\nabla \vec{V}+\nabla \vec{V}^{\mathrm{T}}\right)$ represents the deformation of the fluid while $B=1 / 2\left(\nabla \vec{V}-\nabla \vec{V}^{\mathrm{T}}\right)$ is the vortical part. Mathematically, $\Omega$ is given as:

$$
\begin{aligned}
& \Omega=\frac{\|B\|_{F}^{2}}{\|A\|_{F}^{2}+\|B\|_{F}^{2}}, \\
& \|A\|_{F}^{2}=\operatorname{tr}\left(A^{\mathrm{T}} A\right)=\sum_{i=1}^{2} \sum_{j=1}^{2}\left(A_{i j}\right)^{2}, \\
& \|B\|_{F}^{2}=\operatorname{tr}\left(B^{\mathrm{T}} B\right)=\sum_{i=1}^{2} \sum_{j=1}^{2}\left(B_{i j}\right)^{2} .
\end{aligned}
$$

To avoid undefined cases and for practical purposes, a small value of $\varepsilon=\delta(b-a)_{\max }$ is added to the denominator to Eq. (6). With this criterion, $\Omega=0$ depicts no flow rotation while $\Omega \rightarrow 1$ reveals that the fluid does rigid body rotation. A standard threshold of $\Omega=0.52$ clearly identifies the vortex structures. The $\Omega$-method has been rarely used to identify vortex structures in turbomachines. Zhang et al. [14-16] are among the few that have employed the $\Omega$-criterion to capture the vortex structures in the complex flow fields in turbine machines. They concluded that the advances of the $\Omega$-criterion in vortex identification of cavitation are well elaborated and analyzed. Furthermore, authors [1, 17-19] have also applied the $\Omega$-criterion in other engineering applications. All vortical structures (weak and strong) were identified under a single standard threshold, $\Omega=0.52$.

For purposes of this study, a side channel pump model with a convex blade is considered. Side channel pumps are becoming popular in the field of fluid machinery due to their ability to supply fluids at higher heads compared to centrifugal and axial pumps at the same tip speed [20, 21]. Numerous studies, remarkably Authors [20, 22-26] have been conducted on the complex flow patterns and performance optimization of side channel pumps. Until now, the flow theory underlying the operation of side channel pumps remains a challenge due to the inability to 
fully identify and reveal the evolution mechanism of vortex structures in the turbulent flows. The Reynolds number of the investigated pump based on the mean velocity of the fluid, $v_{1}$ and inlet pipe diameter, $D_{1}$ under design conditions is determined by $R e=\rho \nu_{1} D_{1} / \mu=10^{5}$. Here, $\nu_{1}$ and $D_{1}$ represent the velocity and diameter of the inlet suction pipe respectively. This depicts and confirms the turbulent nature of the flow characterized in side channel pumps [4]. Therefore, this paper mainly presents comparative studies on the robustness and reliability of the newly proposed $\Omega$-criterion against two traditional methods $(Q$ and $\lambda_{2}$ ) to accurately capture the vortex structures in the highly turbulent flows linked to the investigated pump model. Besides, the pressure pulsation and entropy loss distributions characterizing the flows in the pump are comprehensively studied on different planes concerning the vortex structures evolved. The main contribution of this paper is the novel application of $\Omega$-criterion to predict the local flow theory to enable the efficient design of side channel pumps.

\section{Numerical Methodology}

\subsection{Pump Model Description}

Wei et al. [27] first demonstrated the necessity of the convex blade at the outer radius. The unsteady pressure pulsating within the pump significantly reduced with the outer radius convex blade. However, in this study, the side channel pump model replicated for this study is similar to that used by Wang et al. [4] however, a convex blade is introduced at the inner radius. The convex blade used in the present study has thickness, $b_{2}=2 \mathrm{~mm}$ and positioned at a height, $h=7 \mathrm{~mm}$. The pump operated at a rotational speed of $1500 \mathrm{r} / \mathrm{min}$. The primary design specifications of the investigated side channel pump model are enlisted in Table 1. Figure 1 is a description of the studied side channel pump model showing the impeller, convex blade and side channel.

\subsection{Turbulence Model Flow Solver}

Numerical simulations were conducted using the commercial CFX software. The theoretical background of the flow motion is governed by the Navier-Stokes equations. The equations comprise the conservation of mass and momentum equations. Assuming the fluid used (water at $25^{\circ} \mathrm{C}$ ) is constant and incompressible, and then the equations are given as:

$$
\begin{aligned}
& \frac{\partial u_{i}}{\partial x_{j}}=0 \\
& \rho\left[\frac{\partial \overline{u_{i}}}{\partial t}+\frac{\partial \overline{u_{j} u_{i}}}{\partial x_{j}}\right]=\frac{\partial}{\partial x_{j}}\left[\mu\left(\frac{\partial \overline{u_{i}}}{\partial x_{j}}\right)-\rho \overline{u_{j}^{\prime} u_{i}^{\prime}}\right]-\frac{\partial \bar{p}}{\partial x_{i}},
\end{aligned}
$$

Table 1 Specifications of the side channel pump model

\begin{tabular}{ll}
\hline Parameter & Value \\
\hline Impeller outer diameter, $d_{2}(\mathrm{~mm})$ & 150 \\
Impeller inner diameter, $d_{1}(\mathrm{~mm})$ & 80 \\
Blade length, $/(\mathrm{mm})$ & 35 \\
Blade width, $w(\mathrm{~mm})$ & 15 \\
Blade thickness, $b_{1}(\mathrm{~mm})$ & 2 \\
Suction angle, $\theta\left({ }^{\circ}\right)$ & 10 \\
Blade number, $Z$ & 24 \\
Clearance, $s(\mathrm{~mm})$ & 0.2 \\
Side channel outer diameter, $d_{2, s c}(\mathrm{~mm})$ & 150.4 \\
Side channel inner diameter, $d_{1, s c}(\mathrm{~mm})$ & 80 \\
Side channel radius, $\uparrow(\mathrm{mm})$ & 17.6 \\
Wrapping angle, $\boldsymbol{\varphi}\left({ }^{\circ}\right)$ & 30 \\
Convex blade thickness, $b_{2}(\mathrm{~mm})$ & 2 \\
Convex blade height, $h(\mathrm{~mm})$ & 7 \\
\hline
\end{tabular}

where $u_{i}$ and $u_{j}$ represent the absolute and relative velocity components respectively.

The pressure and density of the fluid are denoted by $p$ and $\rho$, respectively. $\mu$ defines the kinematic viscosity measured in $\mathrm{m}^{2} / \mathrm{s}$. $-{\overline{u_{j}^{\prime} u_{i}^{\prime}}}^{\prime}$ is the Reynold stress tensor and defined as:

$$
-\overline{u_{j}^{\prime} u_{i}^{\prime}}=\mu_{t}\left(\frac{\partial \overline{u_{j}}}{\partial x_{i}}+\frac{\partial \overline{u_{i}}}{\partial x_{j}}\right)-\frac{2}{3} k \delta_{i j},
$$

where $k$ is the turbulent kinetic energy, and $\delta_{i j}$ represent the Kronecker delta.

In the present study, the Navier-Stokes equations were closed by the shear stress transport (SST) $k-\omega$ turbulence model to solve the turbulence scales generated in the investigated pump [22, 28]. This turbulence model was developed by Menter [29, 30]. Owing to the robust ability of the SST $k-\omega$ model to combine the $k-\omega$ model in the wall boundary region and $k-\varepsilon$ model in the main stream flow region. The formulations of the SST $k-\omega$ model are provided as:

$k-\omega$ model

$$
\frac{\partial(\rho k)}{\partial t}+\frac{\partial}{\partial x_{j}}\left(\rho U_{j} k\right)=\frac{\partial}{\partial x_{j}}\left[\left(\mu+\frac{\mu_{t}}{\sigma_{k 1}}\right) \frac{\partial k}{\partial x_{j}}\right]+P_{k}-\beta^{\prime} \rho k \omega,
$$

$$
\begin{aligned}
& \frac{\partial(\rho \omega)}{\partial t}+\frac{\partial}{\partial x_{j}}\left(\rho U_{j} \omega\right) \\
& =\frac{\partial}{\partial x_{j}}\left[\left(\mu+\frac{\mu_{t}}{\sigma_{\omega 1}}\right) \frac{\partial \omega}{\partial x_{j}}\right]+\alpha_{1} \frac{\omega}{k} P_{k}-\beta_{1} \rho \omega^{2},
\end{aligned}
$$

Modified $k-\varepsilon$ model 


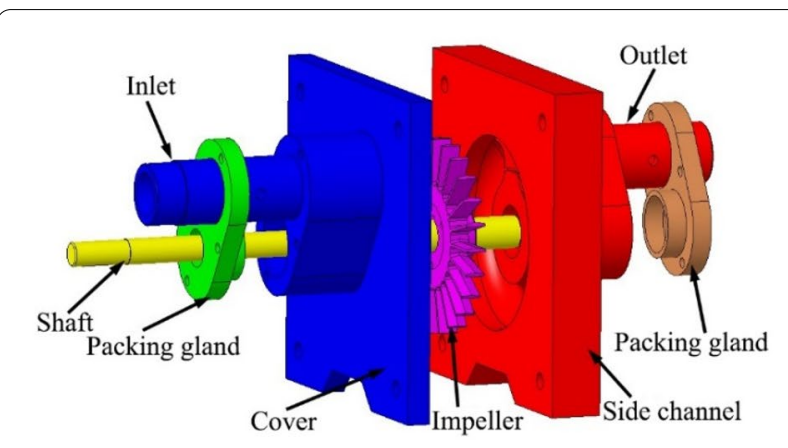

(a)
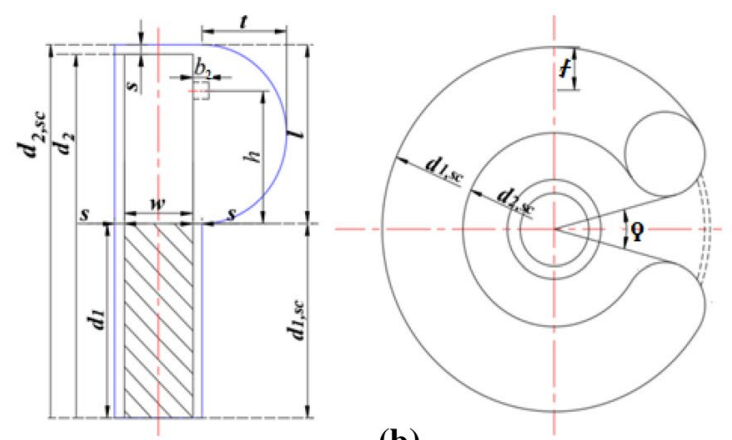

(b)

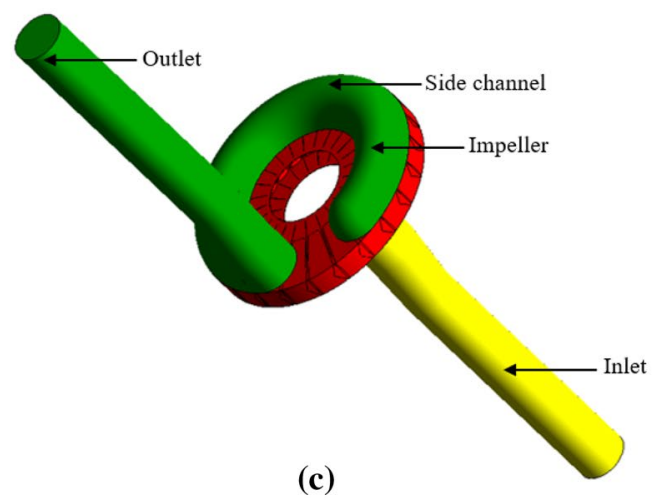

Figure 1 Side channel pump model (a) 3D, (b) 2D, (c) Entire computational domain

$$
\begin{aligned}
& \frac{\partial(\rho k)}{\partial t}+\frac{\partial}{\partial x_{j}}\left(\rho U_{j} k\right)=\frac{\partial}{\partial x_{j}}\left[\left(\mu+\frac{\mu_{t}}{\sigma_{k 2}}\right) \frac{\partial k}{\partial x_{j}}\right]+P_{k}-\beta^{\prime} \rho k \omega, \\
& \frac{\partial(\rho \omega)}{\partial t}+\frac{\partial}{\partial x_{j}}\left(\rho U_{j} \omega\right)=\frac{\partial}{\partial x_{j}}\left[\left(\mu+\frac{\mu_{t}}{\sigma_{\omega 2}}\right) \frac{\partial \omega}{\partial x_{j}}\right] \\
& +2\left(1-F_{1}\right) \rho \frac{1}{\sigma_{\omega 2} \omega} \frac{\partial k}{\partial x_{j}} \frac{\partial \omega}{\partial x_{j}}+\alpha_{2} \frac{\omega}{k} P_{k}-\beta_{2} \rho \omega^{2} .
\end{aligned}
$$

The coefficients of the models of the $k-\omega$ and $k-\varepsilon$ models applied are provided in Table 2.

Total pressure of $1 \mathrm{kPa}$ was used as the inlet boundary condition. The flow direction was normal to the
Table 2 Values of coefficients

\begin{tabular}{llllll}
\hline Coefficients & \multicolumn{6}{c}{} \\
\hline Model & $\boldsymbol{\sigma}_{\boldsymbol{k}}$ & $\boldsymbol{\sigma}_{\boldsymbol{\omega}}$ & $\boldsymbol{\beta}$ & $\boldsymbol{\alpha}$ & $\boldsymbol{\beta}^{\prime}$ \\
\hline$k-\omega(1)$ & 1.176 & 2.0 & 0.075 & $5 / 9$ & 0.09 \\
$k-\varepsilon(2)$ & 1.0 & $1 / 0.865$ & 0.828 & 0.44 & - \\
\hline
\end{tabular}

boundary condition. Opening cylindrical velocity terms were specified at the outlet boundary condition for each operating condition. The results of the steady-state calculations failed to provide accurate flow characteristics and did not agree with the experimental measurements. Therefore, the impeller-side channel interface was updated to transient rotor-stator for the unsteady-state calculation with a time step, $\Delta t=1.111 \times 10^{-4} \mathrm{~s}$. The total time for the unsteady calculations was $2.0 \times 10^{-1} \mathrm{~s}$ for five impeller revolutions. High-resolution advection and the second-order backward Euler transient schemes were recommended to solve the convection and diffusion terms. To ensure numerical accuracy, the residual targets of the continuity, momentum, $k, \omega$ and $\varepsilon$ equations were set at $10^{-4}$.

\subsection{Mesh Domains and Independence Study}

As a requirement for numerical simulations, computational mesh cells were generated using ANSYS ICEM. From Refs. [31-34], the hexahedral structured meshing technique is applied to create cells for the impeller and side channel computational domains. The mesh of the convex blade and clearances (both axial and radial) are created together with the impeller (see Figure 2(b) and (c)). Similarly, the outlet pipe and side channel were meshed as a single domain (see Figure 2(d)) to avoid creating several interfaces which in turn reduces the numerical deviations. Mesh refinement was done at the boundary where the flow exchanges between the impeller and side channel.

To ensure that the simulated flow in the pump is independent of the number of nodes, five different sets of meshes were created with different number of nodes. Table 3 provides a mesh independence study. The head is determined using:

$$
H=\frac{p_{2}-p_{1}}{\rho g} .
$$

Mesh 3 with a suitable topology and number of nodes was chosen and used for the numerical calculations due to the minimal effect of the number of nodes on the head of the pump. Since the mesh size is related to the $Y+$ value, the $Y+$ is an important quantity to determine the non-dimension distance from the wall to the first mesh 


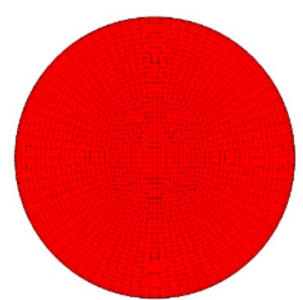

(a)

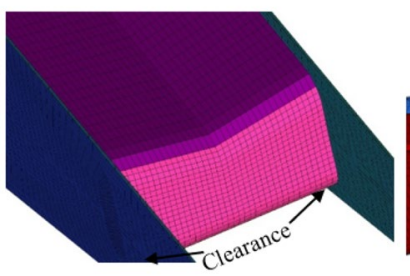

(c)

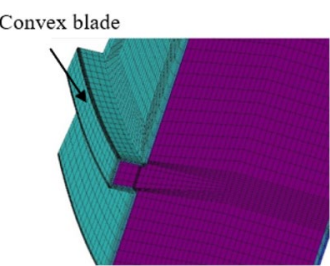

(b)

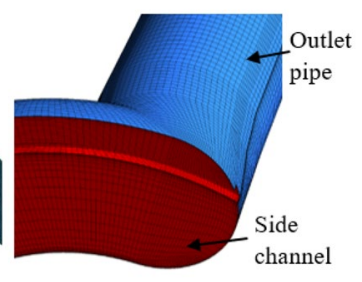

(d)
Figure 2 Mesh domains (a) inlet, (b) impeller, (c) hub of impeller, (d) side channel

Table 3 Mesh independence study

\begin{tabular}{lccccc}
\hline $\begin{array}{l}\text { Item (Number of } \\
\text { nodes) }\end{array}$ & Mesh 1 & Mesh 2 & Mesh 3 & Mesh 4 & Mesh 5 \\
\hline Inlet $\left(\times 10^{6}\right)$ & 0.108 & 0.136 & 0.210 & 0.254 & 0.333 \\
Impeller $\left(\times 10^{6}\right)$ & 0.644 & 1.481 & 2.407 & 3.074 & 4.126 \\
Side channel $\left(\times 10^{6}\right)$ & 0.181 & 0.359 & 0.516 & 0.619 & 0.786 \\
Total $\left(\times 10^{6}\right)$ & 0.933 & 1.976 & 3.133 & 3.947 & 5.245 \\
Head, $H(\mathrm{~m})$ & 11.72 & 10.84 & 10.74 & 10.72 & 10.71 \\
Error $(\%)$ & - & 7.55 & 0.84 & 0.20 & 0.07 \\
\hline
\end{tabular}

node. The averaged $Y+$ values of the impeller and side channel are 13 and 11 respectively. The maximum $Y+$ value is less than 65 for the entire pump, and it is acceptable for the industrial applications. The $Y+$ distribution on the impeller and side channel walls are presented in Figure 3 using the SST $k-\omega$ model. Most regions reveal $Y+$ value below 10 except for some portions of the side channel. Thus, the boundary details of all physical surfaces are set as to automatic non-slip wall function with smooth wall roughness.

\subsection{Validation of Numerical Model}

The reliability of the numerical simulations was verified by comparing them with the head observed in a closed experimental set-up. Figure 4 shows the head coefficient
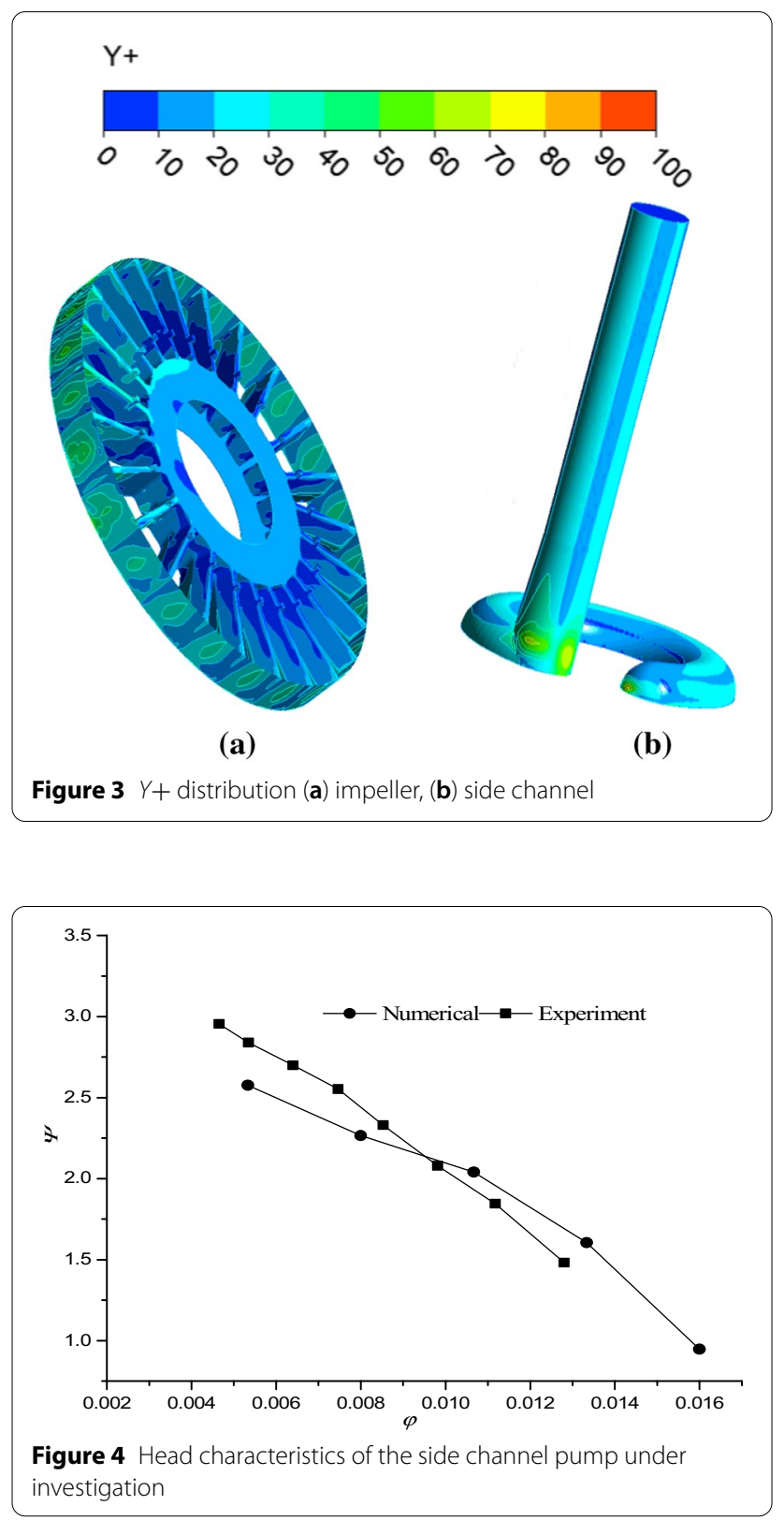

of the investigated side channel pump. The mean flow coefficient, $\varphi$ and head coefficient, $\psi$ were evaluated using the following expressions respectively.

$$
\begin{aligned}
& \varphi=\frac{4 \dot{V}}{\pi^{2} n d_{2}^{3}}, \\
& \psi=\frac{2 g H}{\pi^{2} d_{2}^{2} n^{2}} .
\end{aligned}
$$




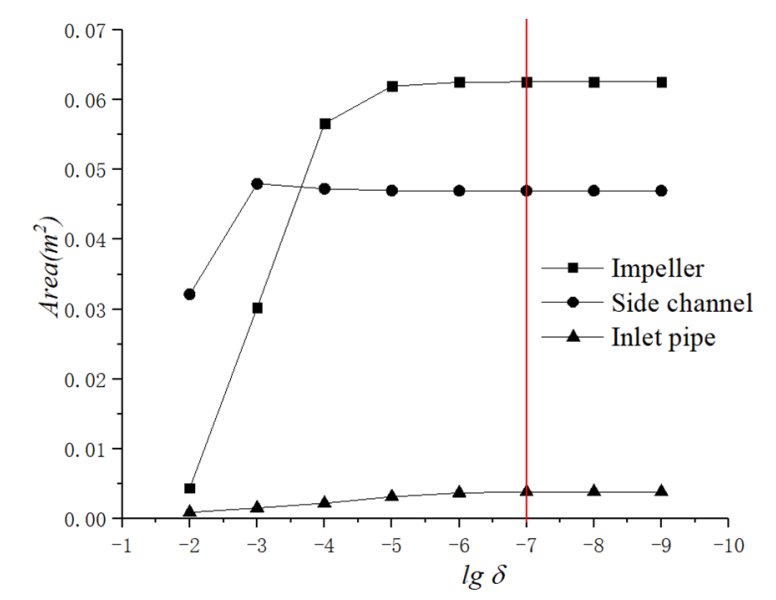

Figure 5 Dependency analysis of $\delta$ value

For the measured operating conditions, the flow coefficient is in a range of $\phi=0.00249$ to 0.016 . Both results from the experiments and numerical approach show a common decreasing tendency from $\psi=0.0032774$ to 0.001484 . A good agreement is established with a maximum relative error of $12.3 \%$ at $\phi=0.0128$ over the experimental measurements. This deviation is partly due to the highly turbulent patterns of the flows and the losses such as mechanical and disk frictional losses that were not considered in the simulation. Therefore, the numerical calculation model used in this study is suitable to predict the complex vortex structures accompanying the flow in the investigated pump.

\section{Results and Discussions}

\subsection{Determination of Appropriate $\delta$ Value for $\Omega$-Criterion \\ in Side Channel Pumps}

It should be noted that the arithmetic average of the transient dataset obtained from the unsteady simulations are used to generate the results for detailed analyses. As pointed out earlier, a small positive value, $\varepsilon$ is introduced to Eq. (6) to achieve the validity of the equation as presented in Eq. (19).

$$
\Omega=\frac{\|B\|_{F}^{2}}{\|A\|_{F}^{2}+\|B\|_{F}^{2}+\varepsilon}=\frac{b}{a+b+\varepsilon},
$$

where $\varepsilon=\delta(b-a)_{\max }$. According to Dong et al. [35], the value of $\varepsilon$ is case dependent due to the computational noises associated with different flows. Importantly, $\varepsilon$ is introduced only to remove the noise but not a threshold to vary the iso-surfaces of the vortex structures. Different values of $\delta$ in the range of $10^{-9} \leq \delta \leq 10^{-2}$ are chosen to determine the influence of $\varepsilon$ in $\Omega$-criterion applications in side channel pumps. Figure 5 examines the dependency of $\delta$ on the value of $\varepsilon$ on inlet, impeller and side channel domains of the pump. Here, the area here is the proportion of the iso-surfaces of the vortex structures formed in each domain. Generally, the flow characteristics in the impeller account for the largest number of vortex structures generated in the entire pump. When $\lg \delta \leq 10^{-7}$, there is minimal or no change in the value of the area for all domains thus no significant effect on the $\varepsilon$ value is observed after $\delta=10^{-7}$. The deviation between the area of the impeller and side channel is $24.98 \%$. The error defined by Eq. (20) at $\delta=10^{-7}$ is the same for the impeller and side channel domains at $0.014 \%$. The error recorded for the inlet pipe is $0.47 \%$.

$$
\Delta \text { error }=\frac{A r e a_{\lg \delta}-A r e a_{\lg \delta-1}}{A r e a_{\lg \delta}} .
$$

The iso-surfaces of the vortex structures formed under different $\delta$ values are presented in Figure 6 . The strength of the vortex structures was colored by the velocity. Ambiguous vortex structures are revealed in the impeller passage when $\delta=10^{-3}$. This observation is counter to Figure 5 where the impeller records the largest area of the vortex structure iso-surfaces. On the other hand, no distinct differences can be recognized between the structures generated at $\delta=10^{-5}$ and $\delta=10^{-7}$. Thus, from both qualitative and quantitative analyses, the small positive parameter is obtained using $\varepsilon=10^{-7}(b-a)_{\max }$ for the $\Omega$-criterion in this case.

\subsection{Comparison of $\Omega$ against $Q$ and $\lambda_{2}$ Criteria}

The operation of side channel pumps leads to various types of vortex structures in the internal flow field. Therefore, the iso-surfaces of the aforementioned vortex criteria are critically applied to accurately identify the vortex patterns in the entire investigated pump domains. Figures 7, 8 and 9 compare the vortex structures under different thresholds for $Q, \lambda_{2}$ and $\Omega$ criteria respectively. All three criteria revealed and confirmed that flow develops a helical pathway after several flow exchanges

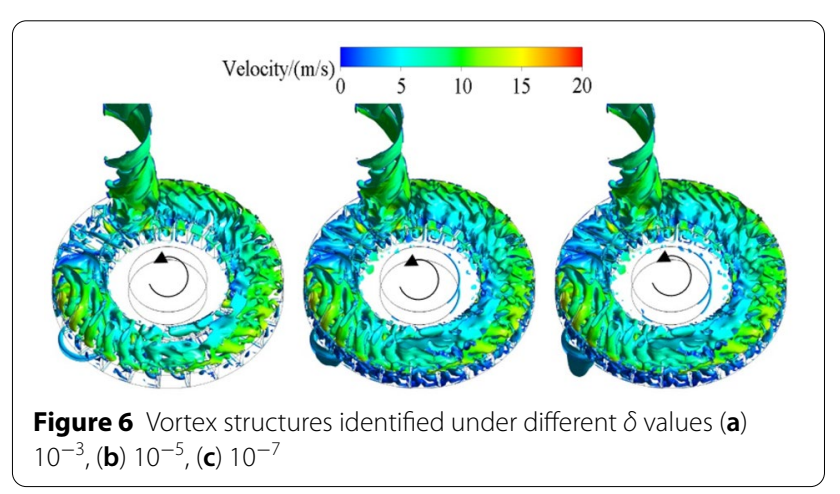




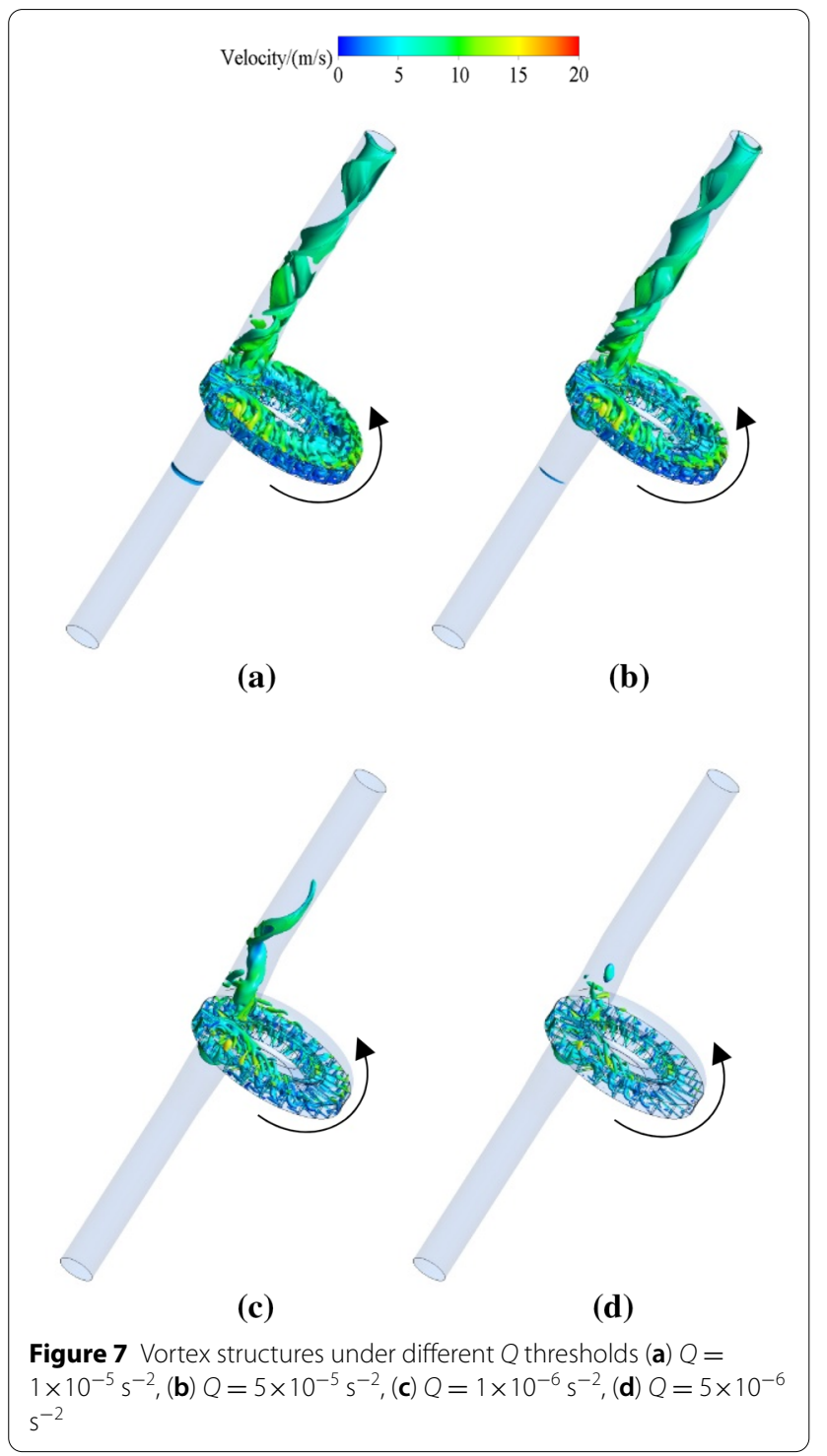

between the impeller and side channel [23]. Notably, this is an important observation since the strength of the exchange induces the several vortex structures produced. With $Q$-criterion, as the threshold value decreases (from $Q=1 \times 10^{-5} \mathrm{~s}^{-2}$ to $Q=5 \times 10^{-6} \mathrm{~s}^{-2}$ ), only weak vortex structures are identified. The $\lambda_{2}$-criterion renders a similar tendency as the threshold value decreases from $\lambda_{2}=-5 \times 10^{-5} \mathrm{~s}^{-2}$ to $\lambda_{2}=-1 \times 10^{-4} \mathrm{~s}^{-2}$. The tendency emanates from Eq. (5), such that when $Q$ is positive, the eigenvalue $\lambda_{2}$ is usually, and a counter observation will be seen if $Q$ is negative [36]. This makes it more challenging to select the suitable threshold using $Q$ and $\lambda_{2}$-criteria. To avoid obscuring some of the vortex structures in the pump, it is essential to choose a standard thresholdbased criterion which is not dependent on the experience of the user. Both strong and weak vortex structures

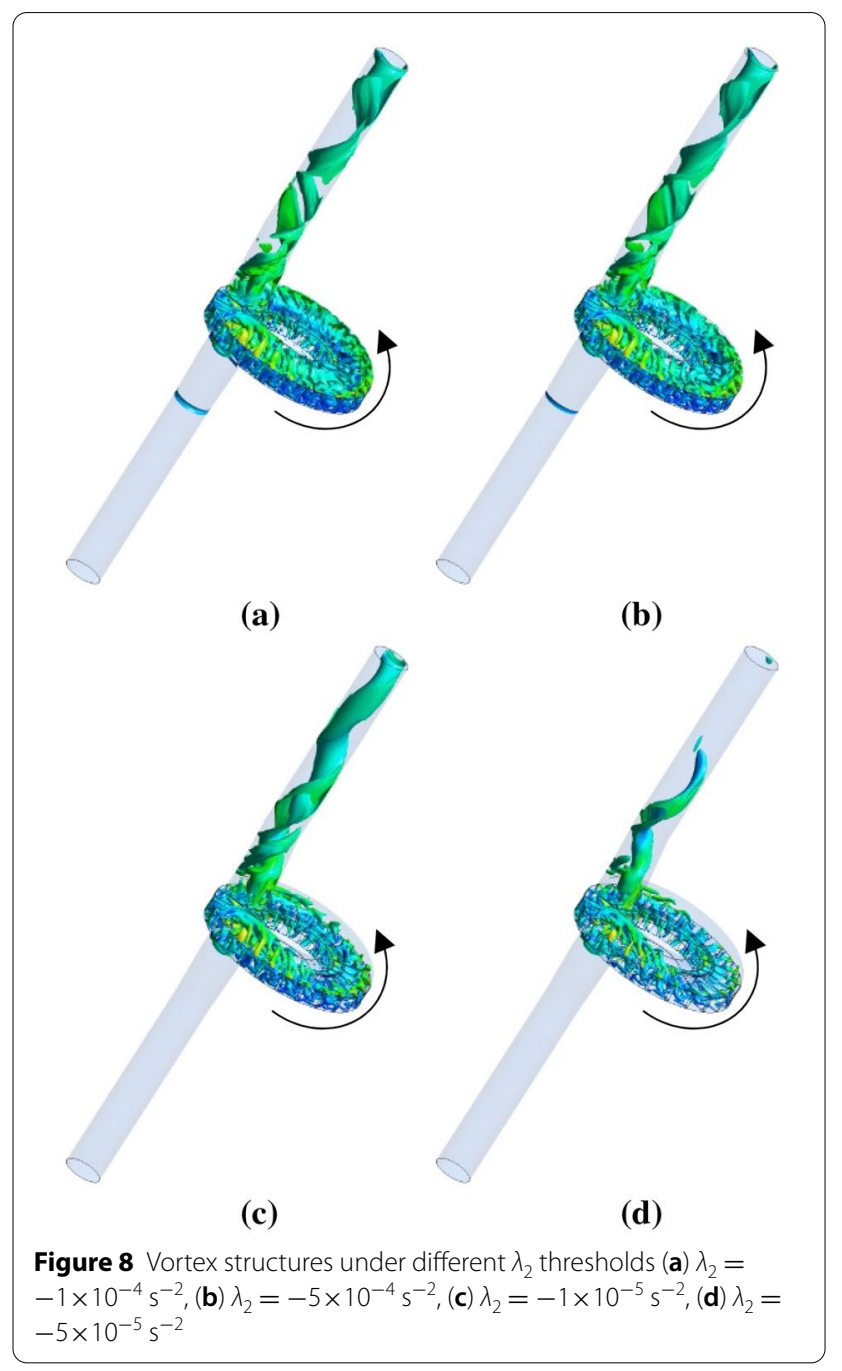

were captured by $\Omega$-criterion when the threshold value was varied from $\Omega=0.52$ to 0.64 . No significant deviations were observed when the threshold changes because vortex is defined as a region where vorticity overtakes deformation. Thus, $\Omega>0.52$ perfectly captures all vortex structures in the entire pump domains. This confirms that amongst the three criteria, $\Omega$ demonstrates less sensitivity to threshold selection. The strength of the velocity distribution is common to the three criteria.

From the earlier observations, $Q$ and $\lambda_{2}$-criteria have confirmed to be threshold-value dependent. Therefore, suitable thresholds of $Q=1 \times 10^{-5} \mathrm{~s}^{-2}$ and $\lambda_{2}=-1 \times 10^{-5} \mathrm{~s}^{-2}$ were chosen to deeply reveal the vortex structures evolved in the impeller passage of the pump. The selection of these threshold values was guided by the results of $\Omega$-criterion with a standard threshold of $\Omega=$ 0.52 . From the inflow, similar trends are seen until the mid-section of the pump for the three criteria (see Figure 10). It is expected that water occupies the impeller 


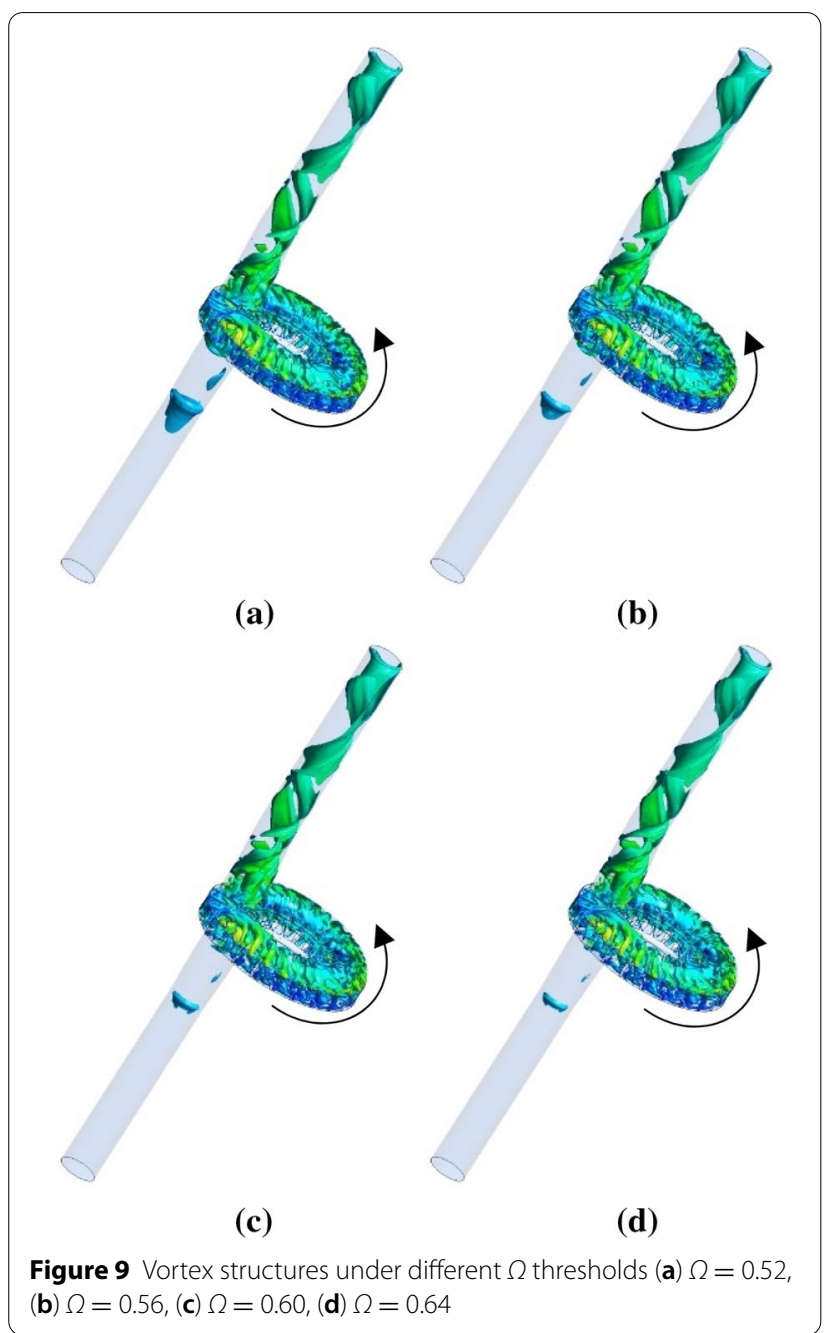

during the flow exchange between the impeller and side channel. For example, at interrupter where there is no channel the impeller passage is fully occupied as identified by the three criteria. The Q-criterion depicts larger empty areas at the inner radius of the blade around the mid-section and closer to the outflow as labeled as $X 1$ and $X 2$ at the same region for the three criteria. A common observation is realized with $\Omega$-criterion but $\lambda_{2}$ reveals otherwise. The inner radius is occupied with the flow at mid-section under $\lambda_{2}$-criteria. It can be assumed that the $\lambda_{2}$-criteria misidentifies the vortex structures at that point (X2) compared to $\Omega$-criterion.

Qualitatively, the iso-surfaces produced from the three criteria in the impeller are in close accordance. However, the orientation of some of the vortex structures illustrates some discrepancies therefore the vortex area under each criterion is plotted as supplied in Figure 11. Guided by the physical definition of $\Omega$-criterion, the area under $\Omega$-criterion is the smallest while $Q$ and $\lambda_{2}$-criteria

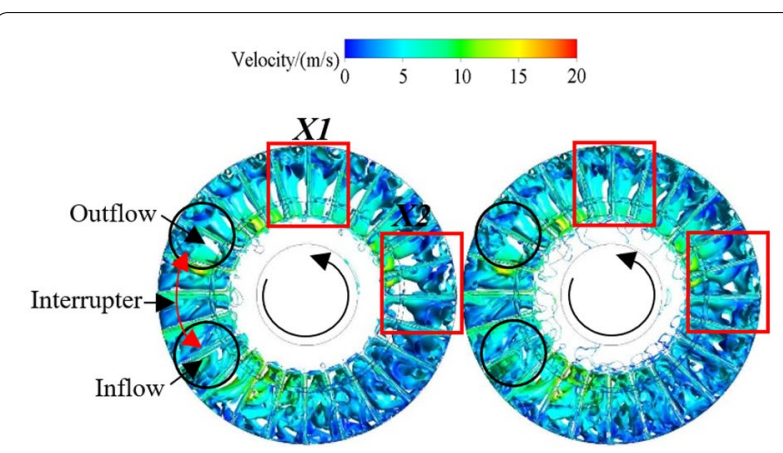

(a)

(b)

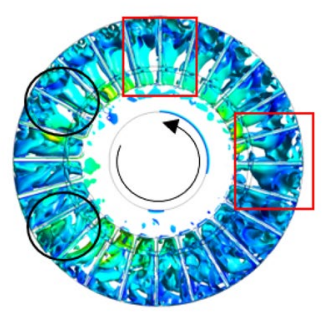

(c)

Figure 10 Vortex structures in the impeller under the three criteria (a) $Q,(\mathbf{b}) \lambda_{2}$ (c) $\Omega$

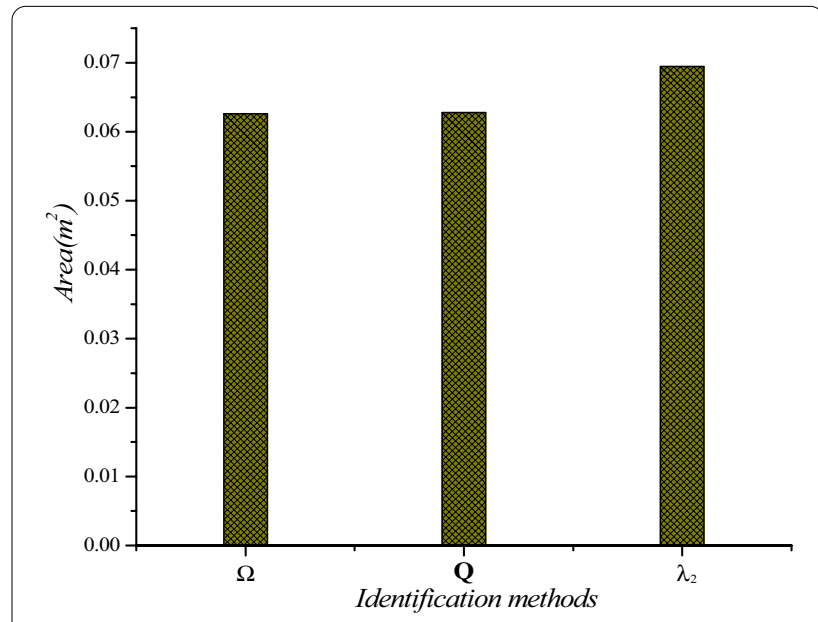

Figure 11 Vortex areas identified under the three criteria

recorded the highest. The area covered under each criterion is expressed as $\Omega, Q$ and $\lambda_{2}$ for the values 0.06262 , 0.06278 and 0.06262 respectively, using the thresholds stated in the paragraph above. The $\Omega$-criterion proves to be reliable and avoids subjective errors during the selection of the threshold.

\subsection{Vortex Structures Intensity under $\Omega$-criterion}

For detailed insight into the vortex evolution and mechanism, different planes (see Figure 12) are set from the 


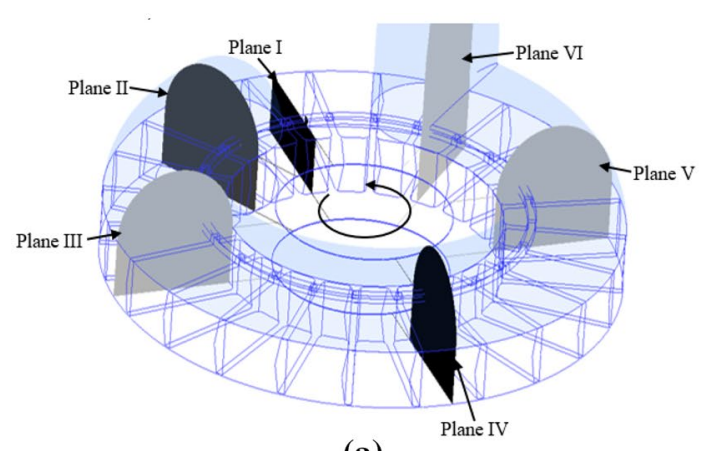

(a)

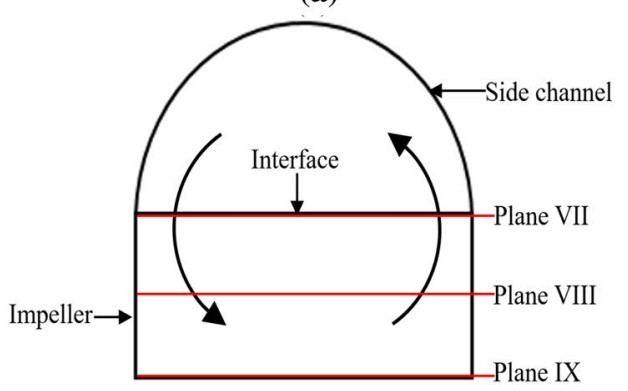

(b)

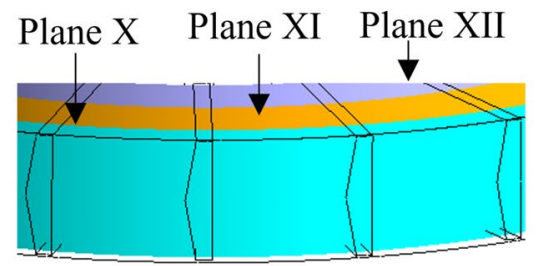

(c)

Figure 12 Positions of different planes (a) longitudinal, (b) axial, and (c) radial

inflow to outflow positions in different directions. Planes I to VI, planes VII to IX and planes X to XII are set in the longitudinal, axial and radial directions respectively. Following previous works [23, 26, 37], a high-performance side channel pump ought to be characterized by strong and orderly organized longitudinal vortices to reduce the flow losses in the impeller passage caused by axial and radial vortices restricted in the impeller. The momentum transfer or exchange of energy of the flow from the impeller to the side channel is responsible for the positive operation (longitudinal vortex)of these kinds of pumps. Typically, the flow is expected to be guided by the outer radius of the impeller into the side channel and re-enter at the inner radius as portrayed (see Figure 12(c)). Side channel pumps mainly dominate three vortex structure types (longitudinal, axial and radial).

The $\Omega$-criterion is non-dimensional and measures from 0 to 1 . According to Liu et al.[13], a vortex is evolved in fluid flow when the threshold is greater than 0.52 . Therefore, values of $\Omega$ greater than 0.8 identify the evolution mechanism and development of a vortex core. In general, the higher the intensity of longitudinal vortex, the more energy the impeller transfers to the water and more work is done. Figure 13 shows the vortex intensity distribution coupled with the velocity vector and streamline plots in the longitudinal direction. There is a number of high vortex intensity regions discovered in the impeller, side channel and as well as the interface. The regions highlighted in black represent the symmetrical longitudinal vortex developed close to the interface. The longitudinal vortex structures promote flow exchange between the impeller and side channel. Furthermore, the axial and radial vortex structures that account for the high vortex intensities are restricted in the impeller passage. These regions are highlighted in red and considered to have negative influence by restraining the longitudinal vortex mechanism. These are in good relation with the convergence of the streamlines.

On plane I which is the interrupter, all the vortex generated are restricted to the impeller due to the absence of the channel. These negatively influenced vortex structures are likely to be transferred to the inflow section of the pump causing undesirable flow patterns. On plane II, the water is received from the inlet pipe, and the flow begins to develop the longitudinal vortex structures as observed in the velocity vector plots due to the weak centrifugal force received. Several vortex structures recognized on this plane are of high intensities greater than 0.65 and constrained in the impeller. This leads to weak longitudinal vortex with interspersed distortions.

As the flow approaches plane III, strong and weak longitudinal vortex structures evolve close to the interface. A vortex core is formed and promotes the flow exchange however some vortex structures restricted close to the impeller impede the longitudinal flow exchange. The velocity vectors and streamlines are in close accordance with the vortex plot. The velocity vectors show uniform and expected flow patterns at plane IV (mid-section). At the mid-section, the flow possesses a significant amount of centrifugal force to enhance the exchange, but the sudden change in geometrical structures restricts some vortex structures in the impeller. The exchange enhances on plane $\mathrm{V}$ with some portions of weak vortex structures in the impeller. Incomplete longitudinal vortex structures are generated close to the interface. At the outflow section VI, the flow leaves the pump with high intensity accounting for the high head built at the outlet pipe.

It is worth noticing that uncharacteristic flow patterns are highlighted in purple on the velocity vector plot on plane VI; instead of the flow to exit at the outer radius, some portions of the flow enter at the outer radius. For plane II, the flow leaves the impeller at the inner radius instead of the outer radius. These 


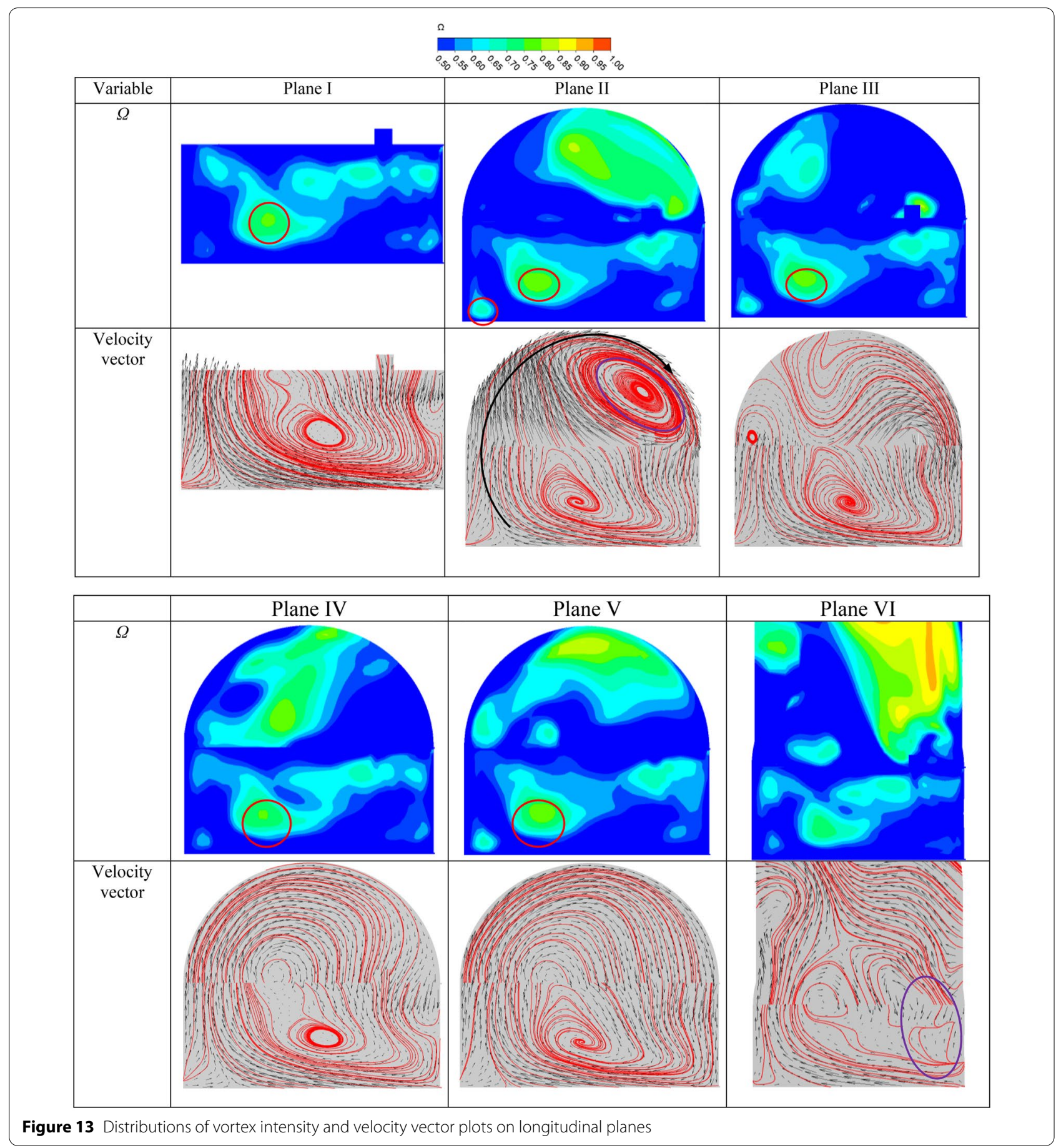

uncharacteristic phenomena lead to violent collisions resulting to a vortex restricted in the impeller. From the above discussions, the intensity of the longitudinal vortex in the longitudinal direction is a little higher but some vortex structures restricted in the impeller relatively generate certain energy losses which affect the generation of longitudinal vortex between the impeller and side channel. The results supplied by the $\Omega$-criterion confirm the physical meaning of vortex as proposed by Liu et al. [13].

As discussed earlier, the vortex structures centered in the impeller are answerable to the low efficiency of side channel pumps because they retard the beneficial effects of the longitudinal vortex. To define the axial vortex in 
the impeller, the total 3D vortex used in the longitudinal plane is reduced to 2D. Here, the velocity gradient tensor $(\nabla \vec{V})$ is presented in a 2D form as $\nabla \vec{V}=\left(\begin{array}{ll}\frac{\partial u}{\partial x} & \frac{\partial u}{\partial y} \\ \frac{\partial v}{\partial x} & \frac{\partial v}{\partial y}\end{array}\right)$. The 2D form is plotted in axial direction at different planes from the base of the impeller to the top close to the interface. Heights of $0.001 \mathrm{~m}, 0.0075 \mathrm{~m}$ and $0.014 \mathrm{~m}$ correspond to planes VII, VIII and IX. Plane VII as shown in Figure 12 is close to the impeller and side channel interface; Plane VIII is set at the middle of the impeller while Plane IX is set at the bottom of the impeller. The velocity vectors and streamlines in the axial planes are also studied per the axial vortex intensity distribution (see Figure 14). The intensity or strength of the axial vortex generally decreases downwards the height of the impeller. On plane VII, high vortex intensities of about $\Omega=0.6$ are identified at the outer radius of each blade pitch except the interrupter. From the velocity vector, secondary and reverse flows are established at the outer radius confirming the results in the axial vortex intensity plots. Sets of axial vortex structures evolve and scatter around the central and inner radiuses of the blade.
On plane VIII, the intensity of the axial vortex formed increases and widely scatters around the inner, central and outer radiuses of the blade. The central radius from visual observation show strong intensity distribution of axial vortex structures. In the middle of the impeller, the velocity vector and streamline plots show that the flow does not gain its full flow characteristics as it moves towards the interface for the exchange with the side channel. Possibly, this could be responsible for the high intensity of axial vortex structures generated. Weak and small amounts of axial vortex structures evolve at the bottom of the impeller close to the pump casing. Noticeably, an axial vortex core develops at central radius on plane VIII. The core increases in size and strength at plane VIII and diminishes on plane IX. Again, the axial vortex structures are concentrated on the pressure side of the blade on all the axial planes.

Figure 15 shows the vortex intensity together with the velocity vectors and streamlines on the radial planes at the mid-section (about $150^{\circ}$ offset from inflow) of the impeller. The radial vortex structures are localized in the impeller by the sides of the blades thereby opposing the momentum exchange developed by the positive

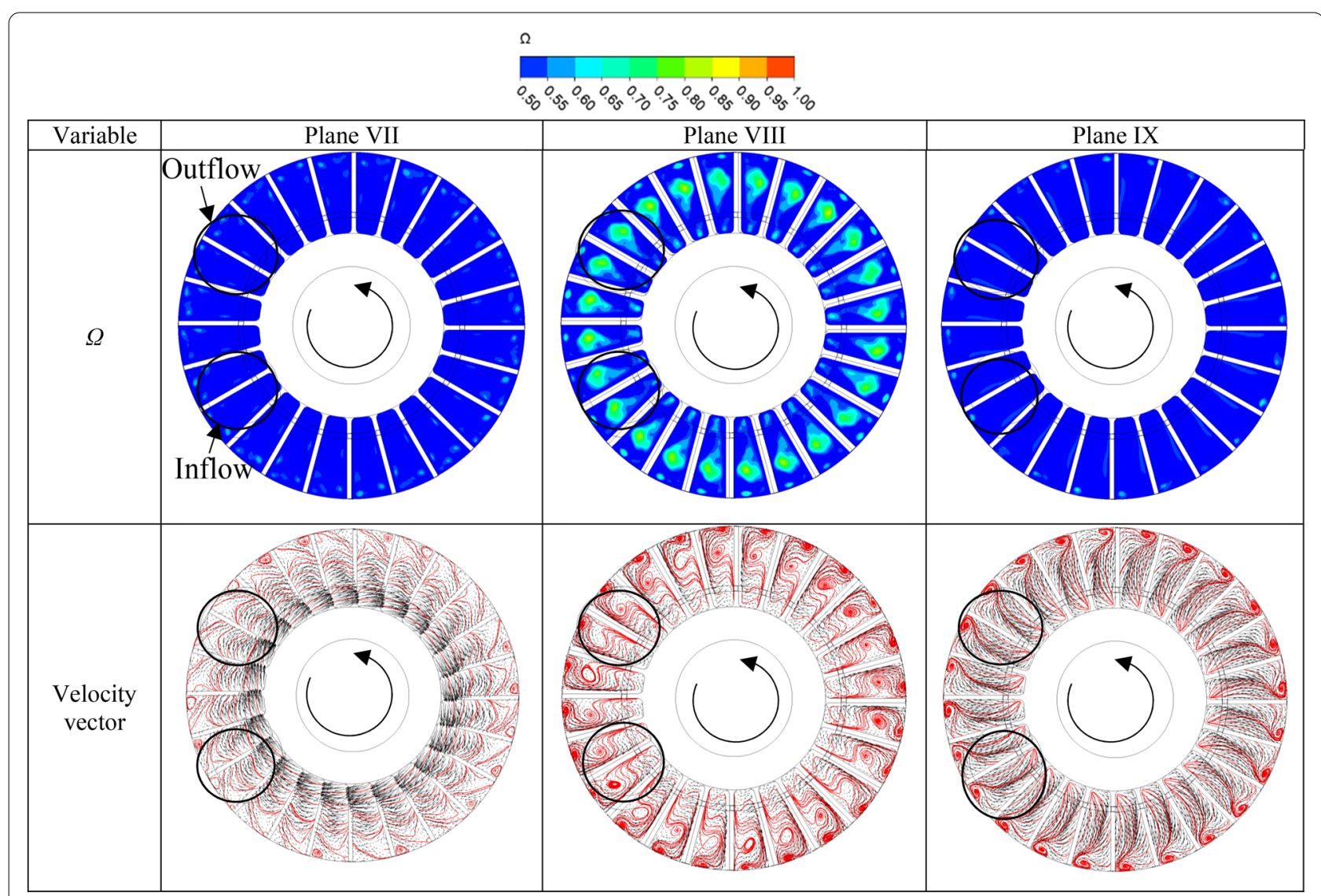

Figure 14 Distributions of axial vortex intensity and velocity vector plots on axial planes 


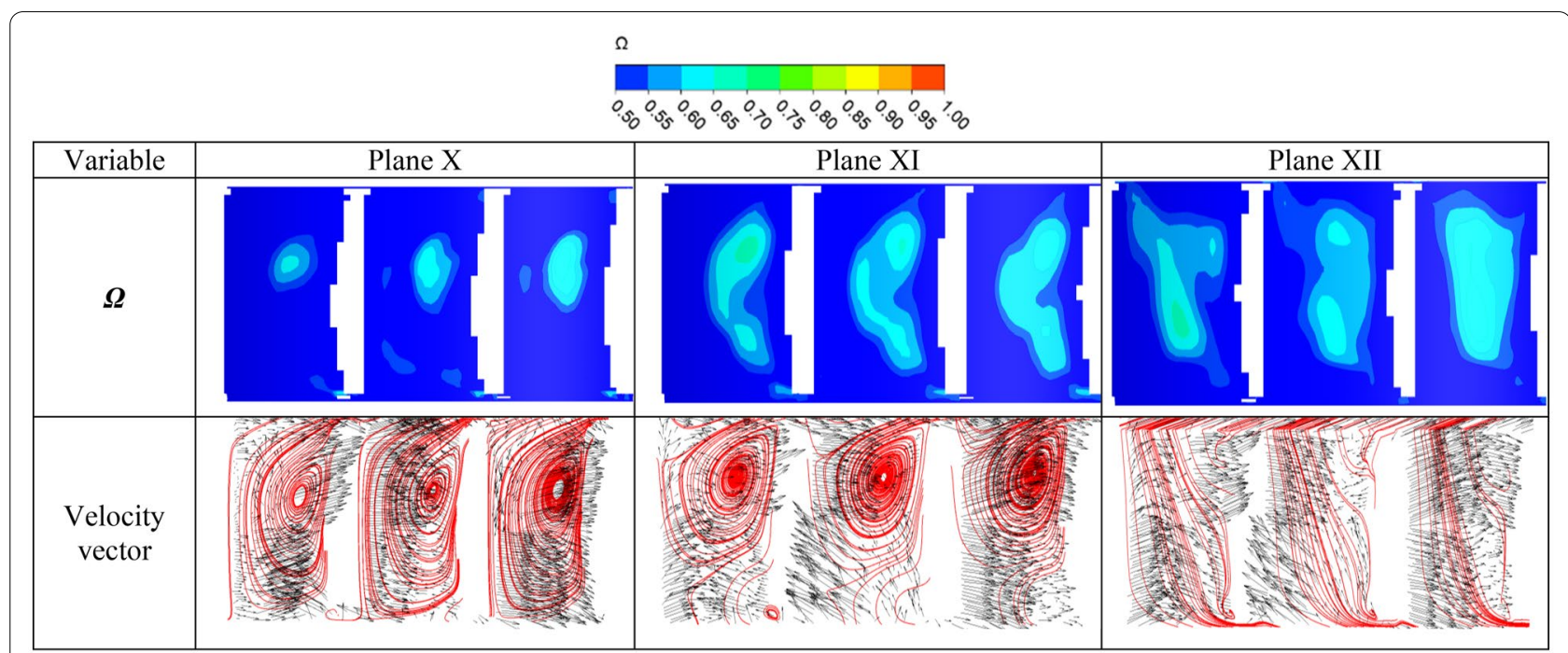

Figure 15 Distributions of vortex intensity and velocity vector plots on radial planes

longitudinal vortex structures. Planes $\mathrm{X}$ to XII are set respectively at radius $0.047 \mathrm{~m}, 0.0575 \mathrm{~m}$ and $0.068 \mathrm{~m}$ from the inner radius to the outer radius. As the flow begins to develop the longitudinal vortex from the inner radius, weak radial vortex structures obstruct this flow action as depicted on the plane X. The middle of the impeller shows the highest $\Omega$ values and the intensity of the radial vortex increases at the central radius on plane XI. A vortex core is developed as highlighted in red. All three blade passages are characterized by radial vortex intensity $\Omega>0.60$. The clearance at the bottom of the impeller shows weak vortex intensity. The convergence of the velocity streamlines perfectly match with some areas of high vortex intensity.

\subsection{Energy Loss Distribution}

For side channel pumps and other hydraulic processes, irreversible losses are associated with their operations. The mechanical energy is always transformed into internal energy owing to the viscosity and highly turbulent flow characteristics. Furthermore, shear can also cause energy dissipation and the energy dissipation partly depends on the negative influences of some vortex structures generated. Therefore, to study the relationship between the energy dissipation and the vortex structures identified by $\Omega$-criterion, the local entropy production method $[38,39]$ provides an effective approach to investigate the negative effects emanating from the highly unsteady flow characteristics in the investigated pump. The turbulent dissipation accounts for the main losses in side channel pumps compared with direct dissipation. Moreover, the impeller generates the highest entropy loss compared to the inlet pipe, side channel and outlet pipe. The mathematical formulations of the entropy production method and their applications in side channel pumps are well explained in Refs. [24, 31] . The coefficient of entropy is defined as:

$$
\xi_{D^{\prime}}=\frac{\Phi_{D^{\prime}} \cdot d_{2}^{3}}{U_{2}^{2} \cdot \rho \cdot \dot{V}}
$$

where $\Phi_{D^{\prime}}$ represents the averaged turbulent dissipation, $U_{2}$ is the circumferential velocity of impeller outlet, $\dot{V}$ is volumetric flow rate, and $d_{2}$ is impeller outer diameter.

As said earlier, the flow enters the pump from the inlet in the circumferential direction on plane II. As the impeller rotates, the flow at the upper part of the inner radius of blade leaks to enter the clearance and rotates along with the impeller. The flow then rotates in the radial direction with the centrifugal force gained. Besides, as the rotational kinetic energy of the impeller on the flow is transformed into pressure head at the outlet, there is backflow from the outflow to the inflow which does not aid in the energy conversion process. From the velocity vector plot, vortex structures along the flow direction are developed in the side channel which induces the flow at the upperpart of the inner radius of the blade. The intensity of the vortex increases to form a vortex core as indicated on the longitudinal vortex plot. This creates high entropy loss, $\xi_{D^{\prime}} \geq 11.0$ at the upper part of the inner radius of the blade as shown in Figure 16. Moreover, the collision as realized on the velocity vector plot in Figure 13 on plane II causes a large vortex which is not characteristic of the pump. Thus, the boundary of the side channel generates high energy loss, $\xi_{D^{\prime}} \geq 11.0$. The 


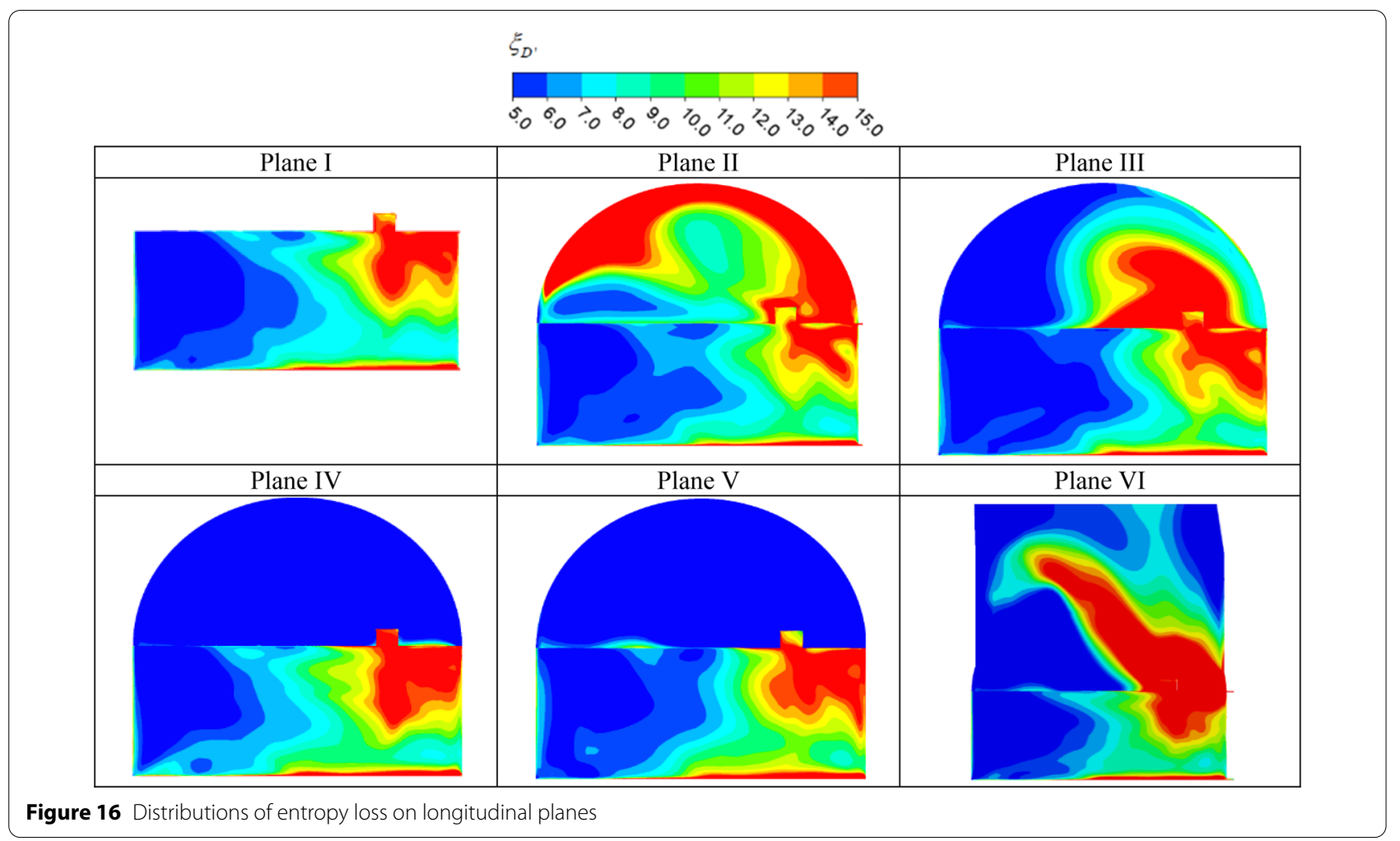

energy loss gradually reduces from plane III in both side channel and impeller. On planes IV and V, the channel records low entropy coefficient of less 7.0 but the losses at the upper part of the inner radius is still evident. Generally, losses produced in the longitudinal plane are mainly located at the inner radius of the blade which affects the output efficiency of side channel pumps. Particularly, the losses located in the impeller are consistent with the vortex structures restricted in the impeller. Planes I to VI show good agreements with the longitudinal vortex plot. On the other hand, the outer radius generates a low loss, $\xi_{D^{\prime}} \leq 7.0$.

Figure 17 presents the entropy loss distribution in the axial planes as referenced in Figure 12. The losses generated in the axial planes are concentrated at the inner radius of both suction and pressure sides of the blade. As

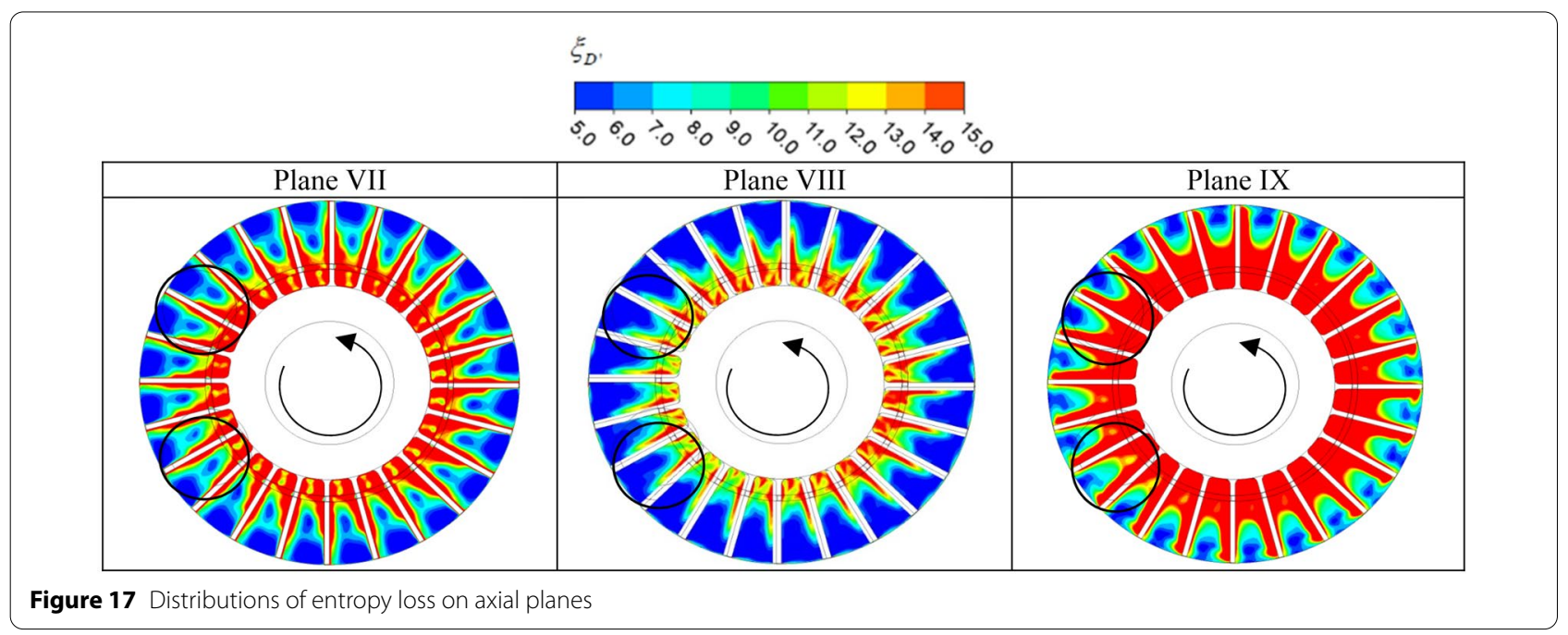


registered on the velocity vector and streamline plots in the axial plane, rotating vortex structures are formed as the flow enters the channel predominately at the inner radius. This weakens the energy output by the impeller to the flow making it distract the flow exchange. Thus, the losses close to the impeller-side channel interface (plane VII) are relatively higher than plane VIII. At the bottom of the impeller, there are high flow leakages that occur at the axial clearance thus efficient longitudinal vortex cannot develop fully to promote the energy transfer process. Therefore, plane IX records the highest $\xi_{D^{\prime}}$ at the inner radius and extends to the central radius as well.

The flow enters the impeller from the inner radius and as shown in the velocity plots in the longitudinal direction, portions of the flow leave at the inner radius causing high vortex cores. The cores partly contribute to the high loss that occurs at the inner radius. Plane $\mathrm{X}$ shows the highest entropy loss as shown in Figure 18. As the flow travels to the outer radius of the blade along the radial direction, the degree of the distribution decreases as the plane is moved from XI to XII. The vortex structures identified in the radial direction show a larger deviation with the entropy loss distribution. The clearance at the bottom of the impeller contributes maximum entropy loss especially on planes $\mathrm{X}$ and $\mathrm{XI}$.

\subsection{Pressure Pulsation Distribution}

As suggested by Zhang et al. [26], the dynamic pressure pulsations in side channel pumps are mainly influenced by the flow exchange between the impeller and side channel. Similarly, the flow exchange brings about the vortex evolution and considering the complex flow characteristics in the investigated pump, and it is important to analyze the pressure pulsations in different planes to the vortex structures identified. The pressure pulsation is monitored for 1800 cycles and the results of the last 24 cycles are examined in this study. The pressure pulsation coefficient is given by the root-mean-square method as:

$$
\begin{aligned}
\bar{p} & =\frac{1}{N} \sum_{i=1}^{N} p_{i}, \\
C_{p}^{\prime} & =\frac{\sqrt{\frac{1}{N} \sum_{i=1}^{N}\left(p_{i}-\bar{p}\right)^{2}}}{0.5 \rho U_{2}^{2}},
\end{aligned}
$$

where $N, p_{i}$ and $\bar{p}$ denote the sample number, the pressure at each time-step, and arithematic average of pressure respectively.

Generally speaking, Figure 19 reveals that the contour of pressure pulsation coefficient; $C_{p}^{\prime}$ in the impeller is higher than the side channel on the longitudinal plane. The impeller reported $C_{p}^{\prime}>0.7$ whiles the side channel also reported $C_{p}^{\prime} \leq 0.7$. With the development of the vortex structures confined in the impeller, it is found that high $C_{p}^{\prime}$ are obvious at the upperparts and roots of the inner radius of the blade on planes I to VI. The intensity of the pressure pulsation at the root of the impeller at the inner radius even reaches the maximum value at all planes in the longitudinal direction. Therefore, the vortex cores developed in the impeller generates significant pulsations.

Figure 20 shows that the distribution of the pressure pulsations intensity on the axial planes in the impeller is high and irregular due to flow turbulence from the vortex structures evolved. Most areas on plane VII reveal $C_{p}^{\prime}>0.7$. The average $C_{p}^{\prime}$ decreases from planes VII to plane VIII but increases on plane IX. Plane IX records maximum $C_{p}^{\prime}$ values at the inner radius of the blade from the inflow to outflow. This could be attributed to the vortex structures generated close to the bottom of the impeller which is partly due to the flow leakage into the axial clearance. By and large, the outer radius showed high $C_{p}^{\prime}$ values compared to the inner radius on plane VII around the mid-section. Maximum values of $C_{p}^{\prime}$ are registered at the central radius of the blade. On plane VIII, $C_{p}^{\prime}$ values at the central radius remarkably decrease. The distribution of the intensity of pressure pulsations in the impeller is

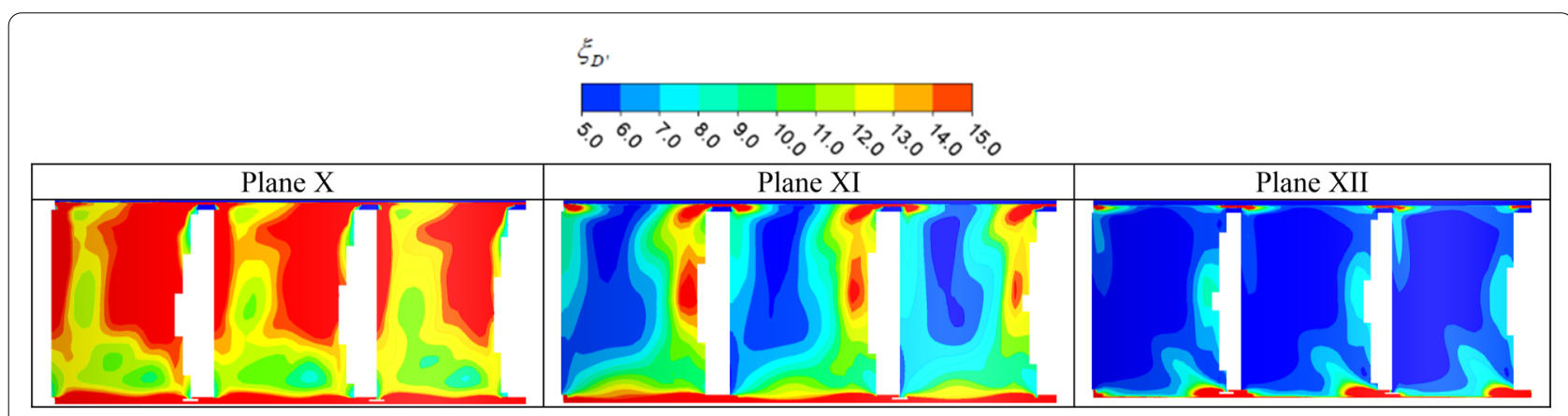

Figure 18 Distributions of entropy loss on radial planes 

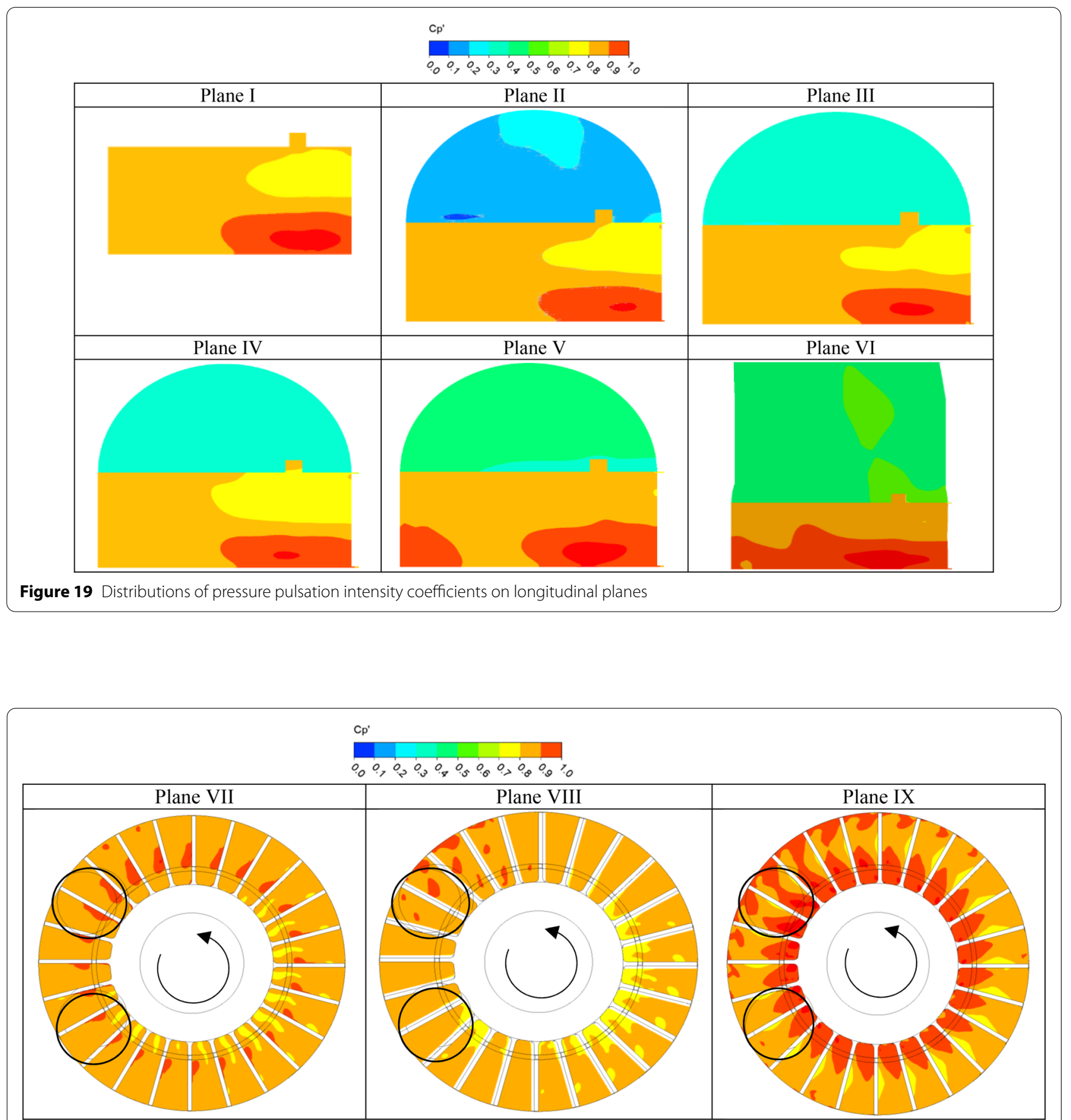

Figure $\mathbf{2 0}$ Distributions of pressure pulsation intensity coefficients on axial planes

generally associated with the flow exchange. The increase in pressure pulsations is conditioned by the weak flow exchange rate in the impeller. Maximum pressure pulsation mostly agrees with the formation of the high vortex core.

The pressure pulsation intensity (see Figure 21) on the radial planes show high $C_{p}^{\prime}$ greater than 0.7 . The root of the blade shows high $C_{p}^{\prime}$ compared with the upperpart on planes X and XI. There is a good agreement between the radial vortex identified and $C_{p}^{\prime}$ distribution on the radial plane. Most regions of high vortex intensity can be recognized with high $C_{p}^{\prime}$ values. 


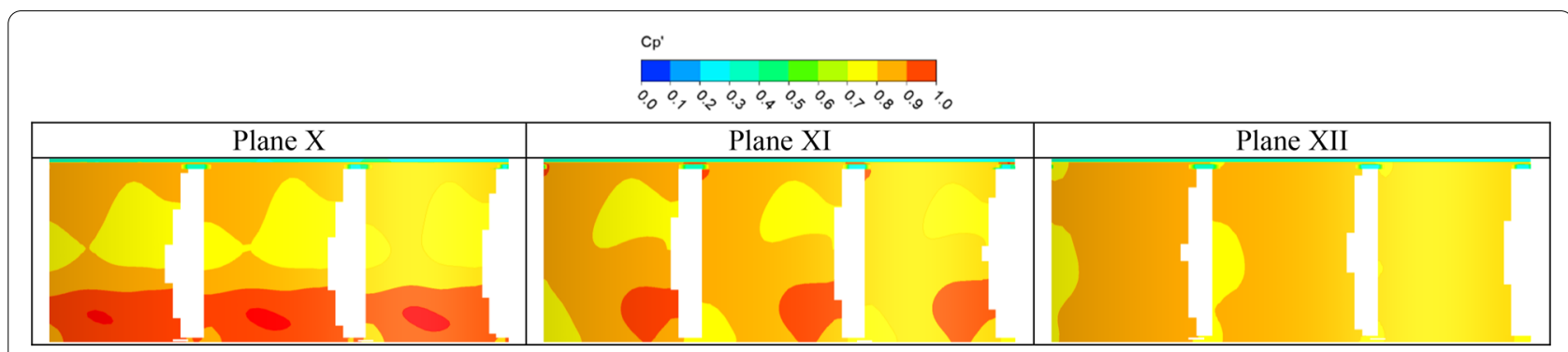

Figure 21 Distributions of pressure pulsation intensity coefficients on radial planes

\section{Conclusions}

The present study elucidates the concept and application of the new omega vortex identification criterion. The criterion provides a clear physical meaning to vortex on the basis that vorticity overtakes deformation in a vortex formation, hence $\Omega=0.52$ approximately describes the vortex boundary. It has several advantages such as the ability to identify both weak and strong vortex structures simultaneously using a standard threshold. The omega criterion was applied to the highly complex 3D flow fields of a side channel pump model after RANS unsteady numerical calculations. Empirically, $\varepsilon$ which is introduced to the denominator of the mathematical definition of the $\Omega$-criterion is expressed as a function which is the maximum of $(b-a) ; \varepsilon=10^{-7}(b-a)_{\max }$. Furthermore, the small parameter, $\varepsilon$ is case-dependent due to the variation of the computational noises associated with different cases, thus the appropriate $\delta$ is obtained for the side channel pump under investigation. After comparing different $\delta$ values in the range of $10^{-9} \leq \delta \leq 10^{-2}, \delta=10^{-7}$ showed minimal change on the iso-surfaces generated by $\Omega$-criterion.

The performance of this criterion was further compared with two traditional and widely used identification criteria, $Q$ and $\lambda_{2}$. The $\Omega$-criterion proves and demonstrates less sensitivity to threshold selection which makes it reliable in the detection of all vortex structures irrespective of the strength using the standard threshold of $\Omega=0.52$. The application of the $\Omega$-criterion to the side channel pump has assisted in the understanding of the vortex structure types associated with the complex flows. The behavior of the vortex structures in comparison with the entropy losses and pressure pulsation distribution showed a fair correlation. The head performance is significantly strengthened by the longitudinal vortex enhancing the momentum exchange between the impeller and side channel. The axial and radial vortex structures restricted in the impeller flow passage impedes the positive momentum exchange rate resulting in high entropy loss and pressure pulsation intensities in the impeller. In conclusion, the high entropy loss and pressure pulsation intensities in the impeller remarkably contribute to the poor efficiency performance of side channel pumps.

\section{Abbreviations}

RANS: Reynolds-averaged Navier-Stokes; SST: Shear stress transport; 3D: Three dimensional.

\section{Nomenclature}

g: Acceleration due to gravity, $\mathrm{m} / \mathrm{s}^{2} ; \bar{p}$ : Arithematic average of pressure, $\mathrm{Pa} ;$ : Blade length, $\mathrm{mm}$; Z: Blade number; $w$ : Blade width, $\mathrm{mm} ; b_{1}$. Blade thickness, $\mathrm{mm} ; F_{i}$ : Blending or auxiliary function in turbulence model; $U_{2}$ : Circumferential velocity of impeller outlet, $\mathrm{m}^{2} / \mathrm{s}$; $\mathrm{s}$ : Clearance, $\mathrm{mm} ; h$ : Convex blade height, $\mathrm{mm} ; b_{2}$ : Convex blade thickness, $\mathrm{mm} ; \rho$ : Density, $\mathrm{kg} / \mathrm{m}^{3} ; \epsilon$ : Dissipation of kinetic energy of turbulence, $\mathrm{m}^{2} / \mathrm{s}^{3} ; \mu$ : Dynamic viscosity, Pa.s; $\xi_{D^{\prime}}$. Entropy loss coefficient; $\varphi$ : Flow coefficient; $H$ : Head, $\mathrm{m} ; \psi$ : Head coefficient; $D_{i}$ Inlet pipe diameter, $\mathrm{mm} ; d_{2}$ : Impeller outer diameter, $\mathrm{mm} ; d_{1}$ : Impeller inner diameter, $\mathrm{mm} ; k$ : Kinetic energy of turbulence, $\mathrm{m}^{2} / \mathrm{s}^{2} ; \delta_{i j}$ : Kronecker's delta; $y^{+}$: Nondimensional wall distance; $p$ : Pressure, Pa; $p_{i}$ : Pressure at each time-step, Pa; $C_{p}^{\prime}$ : Pressure pulsation coefficient; $-\overline{u_{j}^{\prime} u_{i}^{\prime}}$ : Reynolds-stress tensor; $n$ : Rotational speed, $r /$ min; $N$ : Sample number; $d_{2, s c}$ : Side channel outer diameter, $m m ; d_{1, s c}$ : Side channel inner diameter, $\mathrm{mm}$; $\uparrow$ : Side channel radius, $\mathrm{mm}$; $\omega$ : Specific dissipation of turbulence kinetic energy, $1 / \mathrm{s} ; \theta$ : Suction angle, $\left({ }^{\circ}\right)$; $t$ : Time, $s ; \Phi_{D^{\prime}}$ : Turbulent dissipation, $W / \mathrm{m}^{3} ; \beta^{\prime}$ : Turbulence - model coefficients; $\mu_{t}$ : Kinematic viscosity, $\mathrm{m}^{2} / \mathrm{s} ; \sigma_{k}$ : Turbulence - model coefficients; $\sigma_{\omega}$ : Turbulence - model coefficients; : Wrapping angle, $\left({ }^{\circ}\right) ; \dot{V}$ : Volumetric flow rate, $\mathrm{m}^{3} / \mathrm{s} ; u_{i}$ : Velocity components $(u, v, w)$ in Cartesian directions: $x, y, z, m / s ; v i$ Velocity of the inlet suction pipe.

\section{Subscripts \\ $x_{i}:$ : Cartesian coordinates: $x, y, z ; i: j$ : Components in different directions; $x: y, z$ : Coordinates in stationary frame.}

\section{Acknowledgements}

The authors sincerely thanks to Professor Martin Böhle from Technical University of Kaiserslautern for his great support and guidance for this manuscript.

\section{Authors' contributions}

FZ and SY were in charge of the whole study; DA and KC wrote the manuscript; KAA and LZ assisted with sampling and laboratory analyses. All authors read and approved the final manuscript.

\section{Authors' information}

Fan Zhang, born in 1987, is currently an associate professor at National Research Center of Pumps, Jiangsu University, China. His research interest is internal flow in vane pumps. Tel: +86-88780280; E-mail: fzhang@ujs.edu.cn. 
Desmond Appiah, born in 1986, is currently a Ph.D. Post at National Research Center of Pumps, Jiangsu University, China. He is also physics lecturer at University of Education, Winneba, Ghana. E-mail: bembo88donk@yahoo.com Ke Chen, born in 1996, is currently a PhD candidate at National Research Center of Pumps, Jiangsu University, China. E-mail: chenke616161@outlook.com Shouqi Yuan, born in 1963, is currently a full professor at National Research Center of Pumps, Jiangsu University, China. E-mail: shouqiy@ujs.edu.cn Kofi Asamoah Adu-Poku, born in 1988, is currently a PhD candidate at National Research Center of Pumps, Jiangsu University, China. E-mail: adupoku2009@ yahoo.com

Lufeng Zhu, is currently a master candidate at National Research Center of Pumps, Jiangsu University, China. E-mail: 1650454317@qq.com

\section{Funding}

Supported by National Natural Science Foundation of China (Grant Nos. 51809121, 51879121), China Postdoctoral Science Foundation (Grant No. 2021M701535).

\section{Competing Interests}

The authors declare no competing financial interests.

\section{Author Details}

${ }^{1}$ National Research Center of Pumps, Jiangsu University, Zhenjiang 212013, China. ${ }^{2}$ Department of Physics Education, University of Education, Winneba, Ghana.

\section{Received: 4 December 2020 Revised: 30 September 2021 Accepted: 11} November 2021

Published online: 04 December 2021

\section{References}

[1] C Q Liu, J M Liu. Modified normalized Rortex/vortex identification method. Physics of Fluids, 2019, 31(6): 061704.

[2] J Hunt, A A Wray, P Moin. Eddies, streams, and convergence zones in turbulent flows. Studying Turbulence Using Numerical Simulation Databases11, 1988: 193.

[3] J Jeong, F Hussain. On the identification of a vortex. Journal of Fluid Mechanics, 1995, 285: 69-94.

[4] Y F Wang, F Zhang, S Q Yuan, et al. Effect of URANS and hybrid RANSlarge eddy simulation turbulence models on unsteady turbulent flows inside a side channel pump. Journal of Fluids Engineering, 2020, 142(6): 061503.

[5] A V Minakov, D V Platonov, A A Dekterev, et al. The analysis of unsteady flow structure and low frequency pressure pulsations in the high-head Francis turbines. International Journal of Heat and Fluid Flow, 2015, 53: 183-194.

[6] TAsim, R Mishra, B Nsom. Visualisation of turbulent structures in a centrifugal pump's volute using large eddy simulation. 24th Congrès Français de Mécanique, Brest, France, August 26-230, 2019.

[7] T Mihalić, Z Guzović, A Predin, et al. Performances and flow analysis in the centrifugal vortex pump. Journal of Fluids Engineering, 2013, 135(1): 011002-1.

[8] Y Liu, L Tan. Tip clearance on pressure fluctuation intensity and vortex characteristic of a mixed flow pump as turbine at pump mode. Renewable energy, 2018, 129: 606-615.

[9] T Günther, M Schulze, HTheisel, et al. Rotation invariant vortices for flow visualization. IEEE transactions on visualization and computer graphics, 2015, 22(1): 817-826.

[10] H Chang, R K Agarwal, W Li, et al. Numerical and experimental study of a vortex structure and energy loss in a novel self-priming pump. Processes, 2019, 7(10): 701.

[11] Y Liu, L Tan. Spatial-temporal evolution of tip leakage vortex in a mixedflow pump with tip clearance. Journal of Fluids Engineering, 2019, 141 (8): 081302.

[12] Y Liu, L Tan. Theoretical prediction model of tip leakage vortex in a mixed flow pump with tip clearance. Journal of Fluids Engineering, 2020, 142(2): 021203.
[13] C Q Liu, Y Q Wang, Y Yang, et al., New omega vortex identification method. Science China Physics, Mechanics \& Astronomy, 2016, 59(8): 684711.

[14] Y Zhang, K Liu, J Li, et al. Analysis of the vortices in the inner flow of reversible pump turbine with the new omega vortex identification method. Journal of Hydrodynamics, 2018, 30(3): 463-469.

[15] Y Wang, W Zhang, $X$ Cao, et al. The applicability of vortex identification methods for complex vortex structures in axial turbine rotor passages. Journal of Hydrodynamics, 2019, 31(4): 700-707.

[16] CTTran, X Long, B Ji. Vortical structures in the cavitating flow in the Francis-99 draft tube cone under off-design conditions with the new omega vortex identification method. Journal of Physics: Conference Series. IOP Publishing "NTNU, Trondheim, Norway, May 28-29, 2019, 1296(1): 012011.

[17] Y S Gao, C Q Liu. Rortex based velocity gradient tensor decomposition. Physics of Fluids, 2019, 31(1): 011704.

[18] Y Q Wang, Y S Gao, C Q Liu. Galilean invariance of Rortex. Physics of Fluids, 2018, 30(11): 111701.

[19] Y Q Wang, Y Y Yang, G Yang, et al. DNS study on vortex and vorticity in late boundary layer transition. Communications in Computational Physics, 2017, 22(2): 441-459.

[20] D Appiah, F Zhang, S Q Yuan, et al. Effects of the geometrical conditions on the performance of a side channel pump: A review. International Journal of Energy Research, 2017, 42(2): 416-428.

[21] J Pei, M K Osman, W J Wang, et al. Unsteady flow characteristics and cavitation prediction in the double-suction centrifugal pump using a novel approach. Proceedings of the Institution of Mechanical Engineers, Part A: Journal of Power and Energy, 2020, 234(3): 283-299.

[22] A Fleder, M Böhle. A systematical study of the influence of blade length, blade width, and side channel height on the performance of a side channel pump. Journal of Fluids Engineering, 2015, 137(12): 121102

[23] J Pei, F Zhang, D Appiah, et al. Performance prediction based on effects of wrapping angle of a side channel pump. Energies, 2019, 12(1): 139.

[24] F Zhang, D Appiah, F Hong, et al. Energy loss evaluation in a side channel pump under different wrapping angles using entropy production method. International Communications in Heat and Mass Transfer, 2020, 113: 104526.

[25] F Zhang, D Appiah, J F Zhang, et al. Transient flow characterization in energy conversion of a side channel pump under different blade suction angles. Energy, 2018, 161: 635-648.

[26] F Zhang, K Chen, D Appiah, et al. Numerical delineation of 3D unsteady flow fields in side channel pumps for engineering processes. Energies, 2019, 12(7): 1287

[27] XY Wei, F Zhang, D Appiah, et al. Pressure fluctuation reduction in side channel pumps using a modified impeller blade. Fluids Engineering Division Summer Meeting. American Society of Mechanical Engineer, San Francisco, California, USA, July 28-August 1, 2019, AJKFluids: 2019-4932.

[28] F Zhang, A Fleder, M Böhle, et al. Effect of suction side blade profile on the performance of a side channel pump. Proceedings of the Institution of Mechanical Engineers, Part A: Journal of Power and Energy, 2016, 230(6): 586-597.

[29] F Menter. Zonal two equation kW turbulence models for aerodynamic flows. 23rd fluid dynamics, plasmadynamics, and lasers conference. Orlando, USA. July 06-09, 1993. https://doi.org/10.2514/6.1993-2906.

[30] F Menter. Two-equation eddy-viscosity turbulence models for engineering applications. AlAA journal, 1994, 32(8): 1598-1605.

[31] M Böhle, A Fleder, M Mohr. Study of the losses in fluid machinery with the help of entropy. 16th International Symposium on Transport Phenomena and Dynamics of Rotating Machinery, Honolulu, United States, Apr 2016.

[32] A Fleder, M Böhle. Numerical and experimental investigations on the influence of the blade shape on industrial side channel pumps. International Rotating Equipment Conference, 2012.

[33] M Liu, L Tan, S L Cao. Dynamic mode decomposition of gas-liquid flow in a rotodynamic multiphase pump. Renewable Energy, 2019, 139: 1159-1175.

[34] Y Han, L Tan. Dynamic mode decomposition and reconstruction of tip leakage vortex in a mixed flow pump as turbine at pump mode. Renewable Energy, 2020, 155: 725-734. 
[35] X R Dong, Y Q Wang, X P Chen, et al. Determination of epsilon for Omega vortex identification method. Journal of Hydrodynamics, 2018, 30(4): 541-548.

[36] Y L Dong, Y Yang, C Q Liu. DNS study on three vortex identification methods. 55th AIAA Aerospace Sciences Meeting, Grapevine, Texas, January 9-13, 2017, AIAA: 2017-0137.

[37] F Zhang, D Appiah, K Chen, et al. Dynamic characterization of vortex structures and their evolution mechanisms in a side channel pump. Journal of Fluids Engineering, 2020, 142(11): 111502.

[38] F Kock, H Herwig. Entropy production calculation for turbulent shear flows and their implementation in CFD codes. International Journal of Heat and Fluid Flow, 2005, 26(4): 672-680.

[39] F Kock, H Herwig. Local entropy production in turbulent shear flows: a high-Reynolds number model with wall functions. International Journal of Heat and Fluid Flow, 2004, 47(10-11): 2205-2215.

\section{Submit your manuscript to a SpringerOpen ${ }^{\odot}$ journal and benefit from:}

- Convenient online submission

- Rigorous peer review

- Open access: articles freely available online

- High visibility within the field

- Retaining the copyright to your article

Submit your next manuscript at $\boldsymbol{\nabla}$ springeropen.com 